\title{
Efficient updating rough approximations with multi-dimensional variation of ordered data
}

\author{
Shu Wang, ${ }^{\mathrm{a}, \mathrm{b}}$, Tianrui Li $\mathrm{Li}^{\mathrm{a}, \mathrm{b}, *}$, Chuan $\mathrm{Luo}^{\mathrm{c}}$, Hamido Fujita ${ }^{\mathrm{d}}$ \\ ${ }^{a}$ School of Information Science and Technology, Southwest Jiaotong University, Chengdu 611756, China \\ ${ }^{b}$ Key Laboratory of Cloud Computing and Intelligent Technique, Sichuan Province, Chengdu 611756, China \\ ${ }^{c}$ College of Computer Science, Sichuan University, Chengdu 610065, China \\ ${ }^{d}$ Faculty of Software and Information Science, Iwate Prefectural University, 020-0693, Iwate, Japan
}

\begin{abstract}
Ordered data widely exists in practical problems. Dominance-based Rough Set Approach (DRSA) is an effective mathematical tool to obtain approximations of concepts and discovery knowledge from ordered data. In this paper, we focus on the dynamic DRSA for the multi-dimensional variation of an ordered information system, and propose a novel incremental simplified algorithm which can efficiently update approximations of DRSA when objects and attributes increase simultaneously. Most of existing algorithms can efficiently deal with the single-dimensional variation of an information system. However, multi-dimensional variations often occur in real dynamic data. That is, the object set, the attribute set or attribute values of an information system often vary simultaneously. Although we can directly use the definitions of approximations, or integrate some single-dimensional incremental algorithms to cope with multi-dimensional variations, this always results in complex algorithm architectures and a large amount of inefficient computation. In our works, by simplifying traditional definitions of DRSA and employing the incremental learning strategy, we develop an algorithm to neglect unnecessary parameters and avoid much redundant computation. Then, we present two different storage schemes for our proposed algorithm to solve the problem of memory consumption. Finally, a series of experiments are conducted to evaluate its efficiency. Experimental results clearly show that for the two-dimensional variation of objects and attributes in an ordered information system, our proposed algorithm is much faster not only than the non-incremental algorithm based on traditional definitions, but also than the integration of two single-dimensional incremental algorithms.
\end{abstract}

Keywords: Rough sets, Dominance-based rough sets, Incremental learning, Knowledge discovery.

\section{Introduction}

Rough Set Theory (RST) is an excellent mathematical tool for processing vague, imprecise or uncertain problems in reality besides fuzzy set, probability theory and evidence theory [37, 50]. Compared with other uncertainty theories, RST can solve uncertainties by existing data without any priori knowledge. Thus it provides relatively simple interfaces and architectures for data modeling. Recently, RST has been widely applied to many real-world problems in decision making [5, 13, 26, 27], pattern recognition [8, 12, 32, 35, 39, 40, 42] and machine learning [21, 25, 36, 47].

The equivalence relation is defined in classical rough sets to obtain knowledge from an information system. However, for many information systems from practical problems, there usually exists the preference-order relation in their attribute domains and decision classes. These ordered information systems are unable to be processed by classical rough sets. Therefore, Greco et al. defined the dominance relation to substitute the equivalence relation, and proposed the Dominance-based Rough Set Approach (DRSA) [9, 10], which can effectively process the information with preference-order domains. Greco et al. further found that the strict dominance principle in DRSA was unsuitable

\footnotetext{
* Corresponding author.

Email addresses: swang@swjtu.edu.cn (Shu Wang), trli@swjtu.edu.cn (Tianrui Li ), cluo@scu.edu.cn (Chuan Luo), HFujita-799@acm.org (Hamido Fujita)

Preprint submitted to Elsevier
}

August 5, 2016 
to the problems in reality, so they developed a variable-consistency model of DRSA by introducing a consistency level [11]. Kotlowski et al. proposed a probabilistic DRSA model to deal with ordinal classification problems which were monotonously constrained [20]. Blaszczynski et al. found that consistency measures used in the definition of rough approximation lack monotonicity properties, so they proposed a new consistency measure which involves a specific scheme of computation of previous consistency measure within the variable-consistency model of DRSA [2]. Dembczynski et al. reformulated the dominance principle and introduced the novel second-ordered approximations to preserve the properties of approximations, reduce decision tables and induce decision rules [7]. Inuiguchi et al. proposed several variable-precision DRSA to attribute reduction, and showed their properties in comparison with previous DRSA models [16]. For the incomplete decision system, Luo et al. introduced a limited DRSA model to obtain higher accuracies of approximations [34]. Yang et al. successively proposed different DRSA models in incomplete ordered information systems and incomplete interval-valued information systems [45, 46]. They also introduced a new approach for knowledge acquisition in dominance-based incomplete decision systems [44]. Huang et al. presented the notion of dominance intuitionistic fuzzy information systems and established a new dominance relation defined on the score and accuracy function of intuitionistic fuzzy value [14]. Zhang et al. integrated the variable-precision DRSA model with the inclusion measure, and proposed a general framework to study the interval-valued information system [48]. Susmaga introduced a definition of constructs which was context-based in DRSA [43]. Li et al. addressed the problem about constructing the probabilistic rough set model and the multi-granulation decision-theoretic rough set model in the ordered information systems [24]. Ko and Fujita proposed the notion of evidential probability to express the dominance between attributes, and modified DRSA into an evidential model [19]. Besides the theoretical research, many practical applications based on DRSA were also conducted, such as instruments detection [17], airline market management [28-30], business value evaluation [38], and auction mechanisms design [41]. Recently DRSA was further applied to auditing risk judgement [15], candidate supplier selection [15], water quality analysis [18], and vehicle management $[1,15]$.

RST describes uncertain information by two important concepts, namely the upper and lower approximations. Computing approximations is the key step for data mining and knowledge discovery based on rough sets. There are three essential factors in an information system, namely, the object set, the attribute set and attribute values. Dynamic data is certain to lead to variations of the three factors. Therefore, approximations need to be updated in time. According to static algorithms based on the definitions of rough sets, updating approximations is very timeconsuming, because whenever approximations are updated, all data in this information system needs to be processed. If updating approximations can be accelerated, the efficiency of processing data will also be improved. When the object set, the attribute set or attribute values vary individually, many scholars employed the incremental learning strategy and presented a lot of single-dimensional approaches to reduce the time of updating approximations [4, $6,31,33,49]$. For the individual variation of the object set or attribute set in DRSA, Li et al. respectively proposed corresponding single-dimensional incremental algorithms [22, 23]. The algorithm for the variation of objects employs a set-based approach while the algorithm for the variation of attributes employs a matrix-based approach. These two algorithms can efficiently update approximations when objects or attributes are added into an ordered information system. However in real-life problems, the object set or attribute set may vary simultaneously. For example, in instruments detection, when new instruments arrive, they may simultaneously carry new performance indexes which have not been considered before; In water quality analysis, when more water samples are tested, new microorganisms may simultaneously appear, and then corresponding indexes also need to be considered. For these multi-dimensional variations, we may use the definitions to update approximations directly, or we may integrate two single-dimensional algorithms to successively deal with newly added objects and attributes, and then the approximations can also be updated. However, these approaches always result in complex algorithm architectures and inefficient computation. Therefore, the specialized multi-dimensional algorithms for updating approximations of rough sets are needed.

For multi-dimensional variations of an information system, Chen et al. proposed a dynamic two-dimensional algorithm for updating approximations based on the decision-theoretic rough sets when objects and attributes are added simultaneously [3]. They decomposed an information system into different sub-spaces and incrementally updated the equivalence relation in these sub-spaces. They also discussed the approximations of the decision-theoretic rough sets in different levels of granularity. However, there are not any multi-dimensional algorithms for updating approximations of DRSA. Thus in this paper, we manage to design an efficient algorithm to calculate approximations for the simultaneous variation of objects and attributes in DRSA. Our contributions are four-fold: 1) We simplify traditional definitions of DRSA to neglect unnecessary parameters for the updating. 2) We present several new notions, e.g., the 
dominance feature flag, the dominance feature vector and the dominance feature matrix in DRSA, to reflect the dominance relations between objects of their old attributes. By these notions, we employ the incremental learning strategy to avoid much redundant computation. 3) We propose an incremental simplified algorithm for updating approximations efficiently when new objects and attributes are simultaneously added into an ordered information system. 4) We present two implementations for our proposed algorithm based on two different storage schemes to solve the problem of memory consumption. We also conduct a series of experiments in a number of data sets to evaluate their efficiencies.

The remainder of this paper is organized as follows. Firstly we introduce basic concepts of classical rough sets and DRSA in Section 2. In Section 3, we redefine P-generalized decision in DRSA, present the dominance feature flag, the dominance feature vector and the dominance feature matrix, and show their properties. Then two strategies are proposed to accelerate the update of approximations in DRSA with the simultaneous increase of objects and attributes. In Section 4, we design an incremental simplified algorithm based on two different storage schemes for updating approximations for the two-dimensional variation of objects and attributes. Experimental evaluations are conducted in Section 5. Finally, we give the conclusions and our future research directions in Section 6.

\section{Preliminaries}

In this section, we review some concepts, principles of classical rough sets and DRSA [3, 9, 10, 20, 22].

Definition 1. [3] In RST, an information system is denoted by a 4-tuple $S=(U, C \cup D, V, f)$, where

- $U$ is called the universe, which is a nonempty finite set of objects;

- $A=C \cup D, \forall d \in D, C \cap D=\emptyset$. C is a nonempty finite set of condition attributes, and $D$ is a nonempty finite set of decision attributes;

- $V$ is regarded as the domain of all attribute values;

- $f: U \times A \rightarrow V$ is an information function. $\forall a \in A$ and $x \in U$, then $f(x, a) \in V_{a}$, where $V_{a}$ is a domain of attribute a.

In classical rough sets, all objects in the universe are classified by the equivalence relation, but this classification strategy cannot process data with a preference-order relation, while DRSA can overcome this drawback very well. In DRSA, all objects may relate to each other by the preference-order relation with respect to the condition attribute $a$. Such a preference-order relation can be denoted by $\succeq_{a}$. For instance, $\forall x, y \in U, x \geq_{a} y$ means that $x$ is better than or equal to $y$ with respect to the condition attribute $a$.

Definition 2. [9] Let $S=(U, C \cup\{d\}, V, f)$ be an information system. For any nonempty finite attribute set $P \subseteq C$, if $\forall a \in P$ and $x \geq_{a} y$, then $x$ dominates $y$ in $P$, denoted by $x D_{P} y . D_{P}$ is defined to be the dominance relation with respect to $P$.

$$
D_{P}=\{(x, y) \in U \times U: f(x, a) \geq f(y, a), \forall a \in P\} .
$$

On the contrary, if $y \geq_{a} x$, then $x$ is dominated by $y$ in $P$.

According to above definition of the dominance relation, the definitions of dominating and dominated sets are as follows.

Definition 3. [9] Let $S=(U, C \cup\{d\}, V, f)$ be an information system. For any nonempty finite attribute set $P \subseteq C$ and $\forall x \in U$, two important sets of object $x$ are defined :

- P-dominating set : A set of all objects dominating $x$ in $U$.

$$
D_{P}^{+}(x)=\left\{y \in U: y D_{P} x\right\} .
$$


- P-dominated set $:$ A set of all objects dominated by $x$ in $U$.

$$
D_{P}^{-}(x)=\left\{y \in U: x D_{P} y\right\} .
$$

According to the definition of dominance relation $D_{P}$, any object belongs to both of its own $P$-dominating set and $P$-dominated set.

In DRSA, the definition of decision class classified by the decision attribute in an ordered information system is proposed.

Definition 4. [9] Let $S=(U, C \cup\{d\}, V, f)$ be an ordered information system. If the decision attribute $d$ has $m$ different values, then the universe $U$ can be divided into $m$ equivalence classes with respect to the preference-order relation by $d$. This kind of equivalence classes is called the decision class. The set of all decision classes can be denoted as follows:

$$
C l=\left\{C l_{n}, n \in T\right\}, \quad \text { where } T=\{1, \cdots, m\} .
$$

In DRSA, the decision classes are also preference-ordered, thus the sets to be approximated are two united sets instead of the particular classes. These two united sets are called the upward union and downward union respectively, which are defined as follows.

Definition 5. [9] Let $S=(U, C \cup\{d\}, V, f)$ be an ordered information system. If the universe $U$ is divided into $m$ decision classes according to the decision attribute $d$, the upward union $C l_{n}^{\geq}$and the downward union $C l_{n}^{\leq}$are :

$$
C l_{n}^{\geq}=\bigcup_{n^{\prime} \geq n} C l_{n^{\prime}}, C l_{n}^{\leq}=\bigcup_{n^{\prime} \leq n} C l_{n^{\prime}}, \quad \forall n, n^{\prime} \in T, \quad T=\{1, \cdots, m\} .
$$

So $\forall x \in U, x \in C l \geq$ means that $x$ belongs to those decision classes whose labels are larger than or equal to $n$, while $x \in C l_{n}^{\leq}$means that $x$ belongs to those whose labels are less than or equal to $n$.

According to above definitions, the definitions of approximations in DRSA are as follows.

Definition 6. [10] Let $S=(U, C \cup\{d\}, V, f)$ be an ordered information system, for any nonempty finite attribute set $P \subseteq C, n \in T$.

- The lower approximation of the upward union $\mathrm{Cl}_{n}^{\geq}$:

$$
\underline{P}\left(C l_{n}^{\geq}\right)=\left\{x \in U: D_{P}^{+}(x) \subseteq C l_{n}^{\geq}\right\}
$$

- The upper approximation of the upward union $C l_{n}^{\geq}$:

$$
\bar{P}\left(C l_{n}^{\geq}\right)=\left\{x \in U: D_{P}^{-}(x) \cap C l_{n}^{\geq} \neq \varnothing\right\} ;
$$

- The lower approximation of the downward union $\mathrm{Cl}_{n}^{\leq}$:

$$
\underline{P}\left(C l_{n}^{\leq}\right)=\left\{x \in U: D_{P}^{-}(x) \subseteq C l_{n}^{\leq}\right\}
$$

- The upper approximation of the downward union $\mathrm{Cl}_{n}^{\leq}$:

$$
\bar{P}\left(C l_{n}^{\leq}\right)=\left\{x \in U: D_{P}^{+}(x) \cap C l_{n}^{\leq} \neq \varnothing\right\} .
$$

So there are four approximations for the upward and downward unions of each decision class in DRSA.

To show that which intervals of the decision classes an object belongs to, Kotlowski et al. proposed the concept of P-generalized decision [20]. Based on the P-generalized decision, Li et al. proposed related propositions and definitions, by which we can directly compute approximations of DRSA [22]. 
Definition 7. [20] $\forall x \in U$, the P-generalized decision of object $x$ is represented as $\delta_{P}(x)=\left\langle l_{P}(x), u_{P}(x)\right\rangle$, where

$$
\begin{gathered}
l_{P}(x)=\min \left\{n \in T: D_{P}^{+} \cap C l_{n} \neq \varnothing\right\} ; \\
u_{P}(x)=\max \left\{n \in T: D_{P}^{-} \cap C l_{n} \neq \varnothing\right\} .
\end{gathered}
$$

Proposition 1. [22] $\forall x \in U, n \in T$, the followings hold.

(1) If $l_{P}(x) \geq n$, then $x \in \underline{P}\left(C l_{n}^{\geq}\right)$;

(2) If $l_{P}(x) \leq n$, then $x \in \overline{\bar{P}}\left(C l_{n}^{\leq}\right)$;

(3) If $u_{P}(x) \geq n$, then $x \in \bar{P}\left(C l_{n}^{\geq}\right)$;

(4) If $u_{P}(x) \leq n$, then $x \in \underline{P}\left(C l_{n}^{\leq}\right)$.

According to above proposition, $\mathrm{Li}$ et al. redefined the approximations of DRSA as follows:

Definition 8. [22] The approximations of dominance-based rough sets can be represented by the following relations :

$$
\begin{aligned}
& \underline{P}\left(C l_{n}^{\geq}\right)=\left\{x \in U: l_{P}(x) \geq n\right\} ; \\
& \bar{P}\left(C l_{n}^{\geq}\right)=\left\{x \in U: u_{P}(x) \geq n\right\} ; \\
& \underline{P}\left(C l_{n}^{\leq}\right)=\left\{x \in U: u_{P}(x) \leq n\right\} ; \\
& \bar{P}\left(C l_{n}^{\leq}\right)=\left\{x \in U: l_{P}(x) \leq n\right\} .
\end{aligned}
$$

Compared with Definition 6, Definition 8 is based on P-generalized decision to avoid computing the upward and downward unions of decision classes. So the method for updating approximations developed by Definition 8 is simpler than that developed by Definition 6.

\section{Efficient updating approximations in DRSA when objects and attributes increase simultaneously}

With the simultaneous increase of objects and attributes, approximations of DRSA will change and thus need to be updated. To accelerate the updating, we propose two strategies in this section. First, by simplifying the definition of Pgeneralized decision, we no longer need to compute some indirect parameters. Second, by employing the incremental learning strategy, we avoid much unnecessary redundant computation.

\subsection{Simplifying the definition of P-generalized decision}

We start with analyzing the definition of P-generalized decision. According to Definitions 7 and 8 , there are four steps to compute approximations of DRSA by the method based on P-generalized decision:

(1) Computing $D_{P}^{+}(x)$ and $D_{P}^{-}(x)$ of each object $x$ in the universe $U$;

(2) Computing all decision classes $C l_{n}$;

(3) Computing P-generalized decision $l_{P}(x)$ and $u_{P}(x)$ of each object $x$ according to $D_{P}^{+}(x)$ and $D_{P}^{-}(x)$;

(4) Establishing $\underline{P}\left(C l_{n}^{\geq}\right), \bar{P}\left(C l_{n}^{\geq}\right), \underline{P}\left(C l_{n}^{\leq}\right)$and $\bar{P}\left(C l_{n}^{\leq}\right)$according to $l_{P}(x)$ and $u_{P}(x)$.

By analyzing Definitions 7 and 8, we find that the four approximations $\underline{P}\left(C l_{n}^{\geq}\right), \bar{P}\left(C l_{n}^{\geq}\right), \underline{P}\left(C l_{n}^{\leq}\right)$and $\bar{P}\left(C l_{n}^{\leq}\right)$ directly relate to the P-generalized decision values $l_{P}(x)$ and $u_{P}(x)$, while indirectly relate to the dominating set $D_{P}^{+}(x)$ and the dominated set $D_{P}^{-}(x)$ as well as the decision class $C l_{n}$. Thus, if the P-generalized decision values $l_{P}(x)$ and $u_{P}(x)$ can be directly computed only by the dominance relations between objects, and the four approximations $\underline{P}\left(C l_{n}^{\geq}\right), \bar{P}\left(C l_{n}^{\geq}\right), \underline{P}\left(C l_{n}^{\leq}\right)$and $\bar{P}\left(C l_{n}^{\leq}\right)$are established by $l_{P}(x)$ and $u_{P}(x)$, then computing approximations will be simplified and its speed can also be improved.

The two formulae in Definition 7 mean that "the P-generalized decision value $l_{P}(x)$ equals to the minimum label of those decision classes which intersect with the dominating set of object $x$ ", and "the P-generalized decision value 
$u_{P}(x)$ equals to the maximum label of those decision classes which intersect with the dominated set of object $x "$, respectively. Since the label of a decision class is the decision attribute value of an object in this class, the P-generalized decision values $l_{P}(x)$ and $u_{P}(x)$ can be restated as "the minimum decision attribute value of all objects in the dominating set of object $x$ ", and "the maximum decision attribute value of all objects in the dominated set of object $x$ ", respectively. The above descriptions mean that we do not need to compute the decision classes during computing approximations, but to obtain the minimum decision attribute value of the dominating set and the maximum decision attribute value of the dominated set of object $x$. These two values actually are $l_{P}(x)$ and $u_{P}(x)$, respectively.

By the above descriptions, we also find that computing the dominating and dominated sets of object $x$ is unnecessary. The P-generalized decision values $l_{P}(x)$ and $u_{P}(x)$ can be easily obtained by comparing $x$ with other objects in the universe $U$, because $l_{P}(x)$ is in fact the minimum decision attribute value of those objects which are dominating $x$, while $u_{P}(x)$ is in fact the maximum decision attribute value of those objects which are dominated by $x$.

Based on the above analysis, we redefine P-generalized decision as follows:

Definition 9. Let $S=(U, C \cup\{d\}, V, f)$ be an ordered information system, and for any nonempty finite attribute set $P \subseteq C . \forall x \in U$, the $P$-generalized decision of object $x$ is represented as $\delta_{P}(x)=\left\langle l_{P}(x), u_{P}(x)\right\rangle$, where

$$
\begin{aligned}
& l_{P}(x)=\min \left\{f(y, d): y \in U, y D_{P} x\right\} \\
& u_{P}(x)=\max \left\{f(y, d): y \in U, x D_{P} y\right\} .
\end{aligned}
$$

$f(y, d)$ is the decision attribute value of object $y$.

Next, we show that Definition 9 is equivalent to Definition 7.

Proposition 2. $\forall x \in U, \delta_{P}(x)=\left\langle l_{P}(x), u_{P}(x)\right\rangle$ is the P-generalized decision of object $x$, then the following formulae hold.

(1) $l_{P}(x)=\min \left\{n \in T: D_{P}^{+}(x) \cap C l_{n} \neq \varnothing\right\}=\min \left\{f(y, d): y \in U, y D_{P} x\right\}$;

(2) $u_{P}(x)=\max \left\{n \in T: D_{P}^{-}(x) \cap C l_{n} \neq \varnothing\right\}=\max \left\{f(y, d): y \in U, x D_{P} y\right\}$.

Proof. (1) Let $l_{P}(x)=k$, with $k \in T$ and $T=\{1, \cdots m\}$. According to Definition 9 , we have $l_{P}(x)=k=$ $\min \left\{f(y, d): y \in U, y D_{P} x\right\}$. Thus, $\exists y \in U, y \in D_{P}^{+}(x)$ and $y \in C l_{k}$, then $D_{P}^{+}(x) \cap C l_{k} \neq \varnothing$. $\forall n \in T$, if $D_{P}^{+}(x) \cap C l_{n} \neq \varnothing$, then $\exists y, y D_{P} x, y \in C l_{n}$ and $f(y, d)=n$. Since $k=\min \left\{f(y, d): y \in U, y D_{P} x\right\}$, then $k \leq n$. Therefore, $l_{P}(x)=k=\min \left\{n \in T: D_{P}^{+}(x) \cap C l_{n} \neq \varnothing\right\}$.

(2) Let $u_{P}(x)=q$, with $q \in T$ and $T=\{1, \cdots m\}$. According to Definition 9, we have $u_{P}(x)=q=$ $\max \left\{f(y, d): y \in U, x D_{P} y\right\}$. Thus, $\exists y \in U, y \in D_{P}^{-}(x)$ and $y \in C l_{q}$, then $D_{P}^{-}(x) \cap C l_{q} \neq \varnothing$. $\forall n \in T$, if $D_{P}^{-}(x) \cap C l_{n} \neq \varnothing$, then $\exists y, x D_{P} y, y \in C l_{n}$ and $f(y, d)=n$. Since $q=\max \left\{f(y, d): y \in U, x D_{P} y\right\}$, then $q \geq n$. Therefore, $u_{P}(x)=q=\max \left\{n \in T: D_{P}^{-}(x) \cap C l_{n} \neq \varnothing\right\}$.

Compared with the approach based on Definition 7, the approach based on Definition 9 does not need to compute the dominating and dominated sets $D_{P}^{+}(x), D_{P}^{-}(x)$ as well as the decision classes $C l_{n}$ before it gets the P-generalized decision values $l_{P}(x)$ and $u_{P}(x)$. Thus, the revised definition of P-generalized decision greatly simplifies computing approximations in DRSA, so that it can improve the computing efficiency. Following is an example to show the effectiveness of Definition 9.

Example 1. Table 1 illustrates an information system $S=(U, C \cup\{d\}, V, f)$, in which there are preference-order relations between objects, where $U=\left\{x_{1}, x_{2}, \cdots, x_{8}\right\}, C=\left\{a_{1}, a_{2}\right\}, V_{a_{1}}=\{40,55,60,70,80\}, V_{a_{2}}=\{40,50,65,70,80\}$, $V_{d}=\{1,2,3\}$.

Firstly we compute approximations for this information system by Definitions 7 and 8 . Let a nonempty finite attribute set $P=C$, and take an object $x_{1}$ for example. We successively compare the values of two condition attributes $a_{1}$ and $a_{2}$ of object $x_{1}$ with those of other objects in the universe $U$. Then we get the dominating and dominated sets of object $x_{1}$ :

$$
D_{P}^{+}\left(x_{1}\right)=\left\{x_{1}, x_{3}, x_{5}, x_{6}, x_{7}, x_{8}\right\}, \quad D_{P}^{-}\left(x_{1}\right)=\left\{x_{1}, x_{4}\right\}
$$


Table 1: An ordered information system.

\begin{tabular}{llll}
\hline$U$ & $a_{1}$ & $a_{2}$ & $d$ \\
\hline$x_{1}$ & 40 & 65 & 2 \\
$x_{2}$ & 55 & 40 & 1 \\
$x_{3}$ & 60 & 65 & 1 \\
$x_{4}$ & 40 & 50 & 1 \\
$x_{5}$ & 70 & 80 & 2 \\
$x_{6}$ & 80 & 70 & 3 \\
$x_{7}$ & 70 & 70 & 3 \\
$x_{8}$ & 80 & 80 & 3 \\
\hline
\end{tabular}

According to Definition 4, the universe $U$ is divided into three decision classes by the decision attribute $d$ :

$$
C l_{1}=\left\{x_{2}, x_{3}, x_{4}\right\}, \quad C l_{2}=\left\{x_{1}, x_{5}\right\}, \quad C l_{3}=\left\{x_{6}, x_{7}, x_{8}\right\} \text {. }
$$

According to Definition 7, since $D_{P}^{+}\left(x_{1}\right)$ intersects with all of $C l_{1}, C l_{2}$ and $C l_{3}$, we know that the P-generalized decision value $l_{P}\left(x_{1}\right)$ is the minimum label of $C l_{1}, C l_{2}$ and $C l_{3}$, that is, $l_{P}\left(x_{1}\right)=\min \{1,2,3\}=1$. Again $D_{P}^{-}\left(x_{1}\right)$ intersects with $\mathrm{Cl}_{1}$ and $\mathrm{Cl}_{2}$, so $P$-generalized decision value $u_{P}\left(x_{1}\right)$ is the maximum label of these two decision classes, that is, $u_{P}\left(x_{1}\right)=\max \{1,2\}=2$.

Analogously, we can get the dominating and dominated sets of other objects :

$$
\begin{aligned}
& D_{P}^{+}\left(x_{2}\right)=\left\{x_{2}, x_{3}, x_{5}, x_{6}, x_{7}, x_{8}\right\} \\
& D_{P}^{+}\left(x_{3}\right)=\left\{x_{3}, x_{5}, x_{6}, x_{7}, x_{8}\right\} \\
& D_{P}^{+}\left(x_{4}\right)=\left\{x_{1}, x_{3}, x_{4}, x_{5}, x_{6}, x_{7}, x_{8}\right\} \\
& D_{P}^{+}\left(x_{5}\right)=\left\{x_{5}, x_{8}\right\} ; \\
& D_{P}^{+}\left(x_{6}\right)=\left\{x_{6}, x_{8}\right\} ; \\
& D_{P}^{+}\left(x_{7}\right)=\left\{x_{5}, x_{6}, x_{7}, x_{8}\right\} \\
& D_{P}^{+}\left(x_{8}\right)=\left\{x_{8}\right\} \\
& D_{P}^{-}\left(x_{2}\right)=\left\{x_{2}\right\} \\
& D_{P}^{-}\left(x_{3}\right)=\left\{x_{1}, x_{2}, x_{3}, x_{4}\right\} \\
& D_{P}^{-}\left(x_{4}\right)=\left\{x_{4}\right\} ; \\
& D_{P}^{-}\left(x_{5}\right)=\left\{x_{1}, x_{2}, x_{3}, x_{4}, x_{5}, x_{7}\right\} \\
& D_{P}^{-}\left(x_{6}\right)=\left\{x_{1}, x_{2}, x_{3}, x_{4}, x_{6}, x_{7}\right\} \\
& D_{P}^{-}\left(x_{7}\right)=\left\{x_{1}, x_{2}, x_{3}, x_{4}, x_{7}\right\} \\
& D_{P}^{-}\left(x_{8}\right)=\left\{x_{1}, x_{2}, x_{3}, x_{4}, x_{5}, x_{6}, x_{7}, x_{8}\right\}
\end{aligned}
$$

Similarly, we may get the P-generalized decision values of other objects :

$$
\begin{array}{ll}
l_{P}\left(x_{2}\right)=1, & u_{P}\left(x_{2}\right)=1 ; \\
l_{P}\left(x_{3}\right)=1, & u_{P}\left(x_{3}\right)=2 ; \\
l_{P}\left(x_{4}\right)=1, & u_{P}\left(x_{4}\right)=1 ; \\
l_{P}\left(x_{5}\right)=2, & u_{P}\left(x_{5}\right)=3 ; \\
l_{P}\left(x_{6}\right)=3, & u_{P}\left(x_{6}\right)=3 ; \\
l_{P}\left(x_{7}\right)=2, & u_{P}\left(x_{7}\right)=3 ; \\
l_{P}\left(x_{8}\right)=3, & u_{P}\left(x_{8}\right)=3
\end{array}
$$

So according to Definition 8, we obtain approximations of this information system as follows : 


$$
\begin{aligned}
& \underline{P}\left(C l_{1}^{\geq}\right)=U, \quad \underline{P}\left(C l_{2}^{\geq}\right)=\left\{x_{5}, x_{6}, x_{7}, x_{8}\right\}, \quad \underline{P}\left(C l_{3}^{\geq}\right)=\left\{x_{6}, x_{8}\right\} ; \\
& \bar{P}\left(C l_{1}^{\geq}\right)=U, \quad \bar{P}\left(C l_{2}^{\geq}\right)=\left\{x_{1}, x_{3}, x_{5}, x_{6}, x_{7}, x_{8}\right\}, \quad \bar{P}\left(C l_{3}^{\geq}\right)=\left\{x_{5}, x_{6}, x_{7}, x_{8}\right\} ; \\
& \underline{P}\left(C l_{1}^{\leq}\right)=\left\{x_{2}, x_{4}\right\}, \quad \underline{P}\left(C l_{2}^{\leq}\right)=\left\{x_{1}, x_{2}, x_{3}, x_{4}\right\}, \quad \underline{P}\left(C l_{3}^{\leq}\right)=U ; \\
& \bar{P}\left(C l_{1}^{\leq}\right)=\left\{x_{1}, x_{2}, x_{3}, x_{4}\right\}, \quad \bar{P}\left(C l_{2}^{\leq}\right)=\left\{x_{1}, x_{2}, x_{3}, x_{4}, x_{5}, x_{7}\right\}, \quad \bar{P}\left(C l_{3}^{\leq}\right)=U .
\end{aligned}
$$

Below we still take object $x_{1}$ for example to redo the computing by Definition 9. In the universe $U$, objects $x_{1}, x_{3}$, $x_{5}, x_{6}, x_{7}, x_{8}$ are dominating $x_{1}$, and their minimum decision attribute value is 1 . So according to Definition 9, the Pgeneralized decision value $l_{P}\left(x_{1}\right)=1$. On the other side, objects $x_{1}, x_{4}$ are dominated by $x_{1}$. Then the $P$-generalized decision value $u_{P}\left(x_{1}\right)=2$, since the maximum decision attribute value of $x_{1}$ and $x_{4}$ is 2 . Analogously we compute the $P$-generalized decision values of all the other objects as follows :

$$
\begin{array}{ll}
l_{P}\left(x_{2}\right)=1, & u_{P}\left(x_{2}\right)=1 ; \\
l_{P}\left(x_{3}\right)=1, & u_{P}\left(x_{3}\right)=2 ; \\
l_{P}\left(x_{4}\right)=1, & u_{P}\left(x_{4}\right)=1 ; \\
l_{P}\left(x_{5}\right)=2, & u_{P}\left(x_{5}\right)=3 ; \\
l_{P}\left(x_{6}\right)=3, & u_{P}\left(x_{6}\right)=3 ; \\
l_{P}\left(x_{7}\right)=2, & u_{P}\left(x_{7}\right)=3 ; \\
l_{P}\left(x_{8}\right)=3, & u_{P}\left(x_{8}\right)=3 .
\end{array}
$$

Again according to Definition 8 , we obtain approximations of this information system as follows :

$$
\begin{aligned}
& \underline{P}\left(C l_{1}^{\geq}\right)=U, \quad \underline{P}\left(C l_{2}^{\geq}\right)=\left\{x_{5}, x_{6}, x_{7}, x_{8}\right\}, \quad \underline{P}\left(C l_{3}^{\geq}\right)=\left\{x_{6}, x_{8}\right\} ; \\
& \bar{P}\left(C l_{1}^{\geq}\right)=U, \quad \bar{P}\left(C l_{2}^{\geq}\right)=\left\{x_{1}, x_{3}, x_{5}, x_{6}, x_{7}, x_{8}\right\}, \quad \bar{P}\left(C l_{3}^{\geq}\right)=\left\{x_{5}, x_{6}, x_{7}, x_{8}\right\} ; \\
& \underline{P}\left(C l_{1}^{\leq}\right)=\left\{x_{2}, x_{4}\right\}, \quad \underline{P}\left(C l_{2}^{\leq}\right)=\left\{x_{1}, x_{2}, x_{3}, x_{4}\right\}, \quad \underline{P}\left(C l_{3}^{\leq}\right)=U ; \\
& \bar{P}\left(C l_{1}^{\leq}\right)=\left\{x_{1}, x_{2}, x_{3}, x_{4}\right\}, \quad \bar{P}\left(C l_{2}^{\leq}\right)=\left\{x_{1}, x_{2}, x_{3}, x_{4}, x_{5}, x_{7}\right\}, \quad \bar{P}\left(C l_{3}^{\leq}\right)=U .
\end{aligned}
$$

It is obvious that both of the P-generalized decision values and approximations computed based on Definition 9 are identical to those obtained based on Definition 7, but Definition 9 does not compute the dominating and dominated sets as well as the decision classes. Therefore, Definition 9 is simpler and more efficient than Definition 7.

\subsection{Employing the incremental learning strategy}

According to the methods in traditional definitions, whenever new objects and attributes increase simultaneously, we have to recompute the whole information system to obtain updated approximations. The reason is that the dominance relations between objects need to be updated firstly, and the dominance relations are decided by old and new attributes altogether. Here we give an example to demonstrate such case.

Example 2. Table 2 illustrates an ordered information system extended from Table 1. A new object $x_{9}$ and a new attribute $a_{3}$ are simultaneously added into this system. Let $U^{\prime}=U \cup\left\{x_{9}\right\}$ be the updated universe, $C^{\prime}=C \cup\left\{a_{3}\right\}$ be the updated set of condition attributes.

After $x_{9}$ and $a_{3}$ are added into this information system, the dominance relations between objects may change. Unfortunately, we have to compare all the attributes $a_{1}, a_{2}$ and $a_{3}$ again to update the dominance relations between objects. When the data size gets larger, the updating will be fairly time-consuming.

In the above example, if we have known the dominance relations between objects with respect to their old attributes $a_{1}$ and $a_{2}$, then we can get the updated dominance relations incrementally by only comparing the new attribute $a_{3}$. So we define a flag to denote the old dominance relations between objects.

Definition 10. Let $S=(U, C \cup\{d\}, V, f)$ be an ordered information system with $n$ objects in the universe $U . \forall i, j \in$ $\{1,2, \cdots, n\}, x_{i}, x_{j} \in U$. The dominance feature flag $w_{i, j}^{D}$ with respect to objects $x_{i}$ and $x_{j}$ is defined as follows:

$$
w_{i, j}^{D}=\left\{\begin{array}{l}
00, x_{i} \otimes x_{j} \\
01, x_{i}>x_{j} \\
10, x_{i} \prec x_{j} \\
11, x_{i}=x_{j}
\end{array},\right.
$$


Table 2: An ordered information system with a newly added object and a newly added attribute.

\begin{tabular}{lllll}
\hline$U^{\prime}$ & $a_{1}$ & $a_{2}$ & $a_{3}$ & $d$ \\
\hline$x_{1}$ & 40 & 65 & 50 & 2 \\
$x_{2}$ & 55 & 40 & 80 & 1 \\
$x_{3}$ & 60 & 65 & 60 & 1 \\
$x_{4}$ & 40 & 50 & 55 & 2 \\
$x_{5}$ & 70 & 80 & 65 & 3 \\
$x_{6}$ & 80 & 70 & 40 & 3 \\
$x_{7}$ & 70 & 70 & 50 & 3 \\
$x_{8}$ & 80 & 80 & 80 & 2 \\
$x_{9}$ & 80 & 70 & 60 & \\
\hline
\end{tabular}

where,

- $x_{i} \otimes x_{j}: x_{i}$ and $x_{j}$ are dominance independent to each other with respect to the attribute set $C$;

- $x_{i}>x_{j}: x_{i}$ is better than $x_{j}$ with respect to the attribute set $C$;

- $x_{i} \prec x_{j}: x_{i}$ is worse than $x_{j}$ with respect to the attribute set $C$;

- $x_{i}=x_{j}: x_{i}$ equals to $x_{j}$ with respect to the attribute set $C$.

We find that there may be one of the above four possible dominance relations between any two objects $x_{i}$ and $x_{j}$, so four 2-bit binary values are set to distinguish the four relations. For a universe, all objects in it have the dominance feature flags with each others. That is, for a universe with $n$ objects, an object in it has a group of $n$ dominance feature flags. To denote such a flag group, we give the following definition.

Definition 11. Let $S=(U, C \cup\{d\}, V, f)$ be an ordered information system with $n$ objects in the universe $U$. $\forall i \in$ $\{1,2, \cdots, n\}, x_{i} \in U$. The dominance feature vector of object $x_{i}$ is defined as :

$$
V_{i}^{D}=\left(x_{i} w_{i, 1}^{D} w_{i, 2}^{D} \cdots w_{i, n}^{D}\right)
$$

$V_{i}^{D}$ is a row vector. Since each object in the universe carries such a row vector, the feature vectors of all objects organize a matrix just as the following definition shows.

Definition 12. Let $S=(U, C \cup\{d\}, V, f)$ be an ordered information system with $n$ objects in the universe $U$. For $x_{1}, x_{2}, \cdots, x_{n} \in U$, the row vectors $V_{1}^{D}, V_{2}^{D}, \cdots, V_{n}^{D}$ are the dominance feature vectors correspond to $x_{1}, x_{2}, \cdots, x_{n}$ respectively. Then the dominance feature matrix of this information system is defined as :

$$
M^{D}=\left[\begin{array}{c}
V_{1}^{D} \\
V_{2}^{D} \\
\vdots \\
V_{n}^{D}
\end{array}\right]=\left[\begin{array}{ccccc}
x_{1} & w_{1,1}^{D} & w_{1,2}^{D} & \cdots & w_{1, n}^{D} \\
x_{2} & w_{2,1}^{D} & w_{2,2}^{D} & \cdots & w_{2, n}^{D} \\
\vdots & \vdots & \vdots & & \vdots \\
x_{n} & w_{n, 1}^{D} & w_{n, 2}^{D} & \cdots & w_{n, n}^{D}
\end{array}\right]=\left[\begin{array}{ll}
X & W^{D}
\end{array}\right] \text {, }
$$

where, 
$\boldsymbol{X}=\left[\begin{array}{c}x_{1} \\ x_{2} \\ \vdots \\ x_{n}\end{array}\right]$ is the object vector

- $W^{D}=\left[\begin{array}{cccc}w_{1,1}^{D} & w_{1,2}^{D} & \cdots & w_{1, n}^{D} \\ w_{2,1}^{D} & w_{2,2}^{D} & \cdots & w_{2, n}^{D} \\ \vdots & \vdots & & \vdots \\ w_{n, 1}^{D} & w_{n, 2}^{D} & \cdots & w_{n, n}^{D}\end{array}\right]$ is the feature value matrix.

Below we exemplify a practical dominance feature matrix.

Example 3. (Continuation of Example 1)

According to above definitions, we take object $x_{1}$ for example from Table 1 . Then we can get the dominance feature vector of $x_{1}$ :

$$
V_{1}^{D}=\left(x_{1} 1100100110101010\right) .
$$

The dominance feature vectors of other objects in Table 1 can be analogously obtained, and all the dominance feature vectors are arranged row by row according to Definition 12. Then we have the dominance feature matrix of Table 1:

$$
M^{D}=\left[\begin{array}{c}
V_{1}^{D} \\
V_{2}^{D} \\
V_{3}^{D} \\
V_{4}^{D} \\
V_{5}^{D} \\
V_{6}^{D} \\
V_{7}^{D} \\
V_{8}^{D}
\end{array}\right]=\left[\begin{array}{lllllllll}
x_{1} & 11 & 00 & 10 & 01 & 10 & 10 & 10 & 10 \\
x_{2} & 00 & 11 & 10 & 00 & 10 & 10 & 10 & 10 \\
x_{3} & 01 & 01 & 11 & 01 & 10 & 10 & 10 & 10 \\
x_{4} & 10 & 00 & 10 & 11 & 10 & 10 & 10 & 10 \\
x_{5} & 01 & 01 & 01 & 01 & 11 & 00 & 01 & 10 \\
x_{6} & 01 & 01 & 01 & 01 & 00 & 11 & 01 & 10 \\
x_{7} & 01 & 01 & 01 & 01 & 10 & 10 & 11 & 10 \\
x_{8} & 01 & 01 & 01 & 01 & 01 & 01 & 01 & 11
\end{array}\right] .
$$

The above matrix is fairly large, so we manage to reduce its size to save the computer memory. The feature value matrix is in fact regular just as the following proposition shows.

Proposition 3. Let $S=(U, C \cup\{d\}, V, f)$ be an ordered information system with $n$ objects in the universe $U . M^{D}=$ $\left[\begin{array}{ll}X & W^{D}\end{array}\right]$ is the dominance feature matrix of $S$. Then $\forall i, j \in\{1,2, \cdots, n\}, x_{i}, x_{j} \in U$ and $w_{i, j}^{D}, w_{j, i}^{D} \in W^{D}$, the following formula holds.

$$
w_{i, j}^{D}=\left\{\begin{array}{rr}
w_{j, i}^{D}, & x_{i} \otimes x_{j} \text { or } x_{i}=x_{j} \\
\sim w_{j, i}^{D}, & x_{i}>x_{j} \text { or } x_{i}<x_{j}
\end{array},\right.
$$

where, operator “ ” denotes performing a bitwise NOT. And we say that $W^{D}$ is dominance symmetrical.

Since the feature value matrix $W^{D}$ in the above example is dominance symmetrical with respect to its diagonal, we only need the upper triangular part of it. What's more, all its diagonal values must be 11 and thus need not to be kept. Therefore, the dominance feature matrix $M^{D}$ in the above example becomes: 


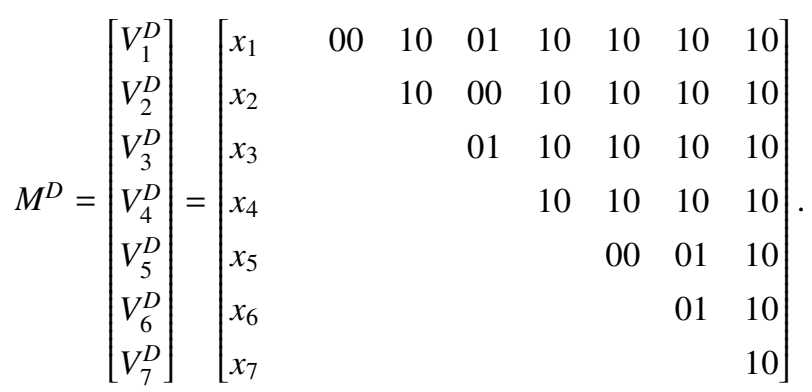

Based on above analysis, we rewrite Definition 11 and Definition 12.

Definition 13. Let $S=(U, C \cup\{d\}, V, f)$ be an ordered information system with $n$ objects in the universe $U . \forall i \in$ $\{1,2, \cdots, n\}, x_{i} \in U$. The dominance feature vector of object $x_{i}$ is defined as:

$$
V_{i}^{D}=\left(x_{i} w_{i, i+1}^{D} w_{i, i+2}^{D} \cdots w_{i, n}^{D}\right) .
$$

Here $V_{i}^{D}$ only records the dominance feature flags which are obtained by comparing $x_{i}$ with those objects behind $x_{i}$ in the universe.

Definition 14. Let $S=(U, C \cup\{d\}, V, f)$ be an ordered information system with $n$ objects in the universe $U$. For $x_{1}, x_{2}, \cdots, x_{n} \in U$, the row vectors $V_{1}^{D}, V_{2}^{D}, \cdots, V_{n-1}^{D}$ are the dominance feature vectors correspond to $x_{1}, x_{2}, \cdots, x_{n-1}$ respectively. Then the dominance feature matrix of this information system is defined as:

$$
M^{D}=\left[\begin{array}{c}
V_{1}^{D} \\
V_{2}^{D} \\
\vdots \\
V_{n-1}^{D}
\end{array}\right]=\left[\begin{array}{ccccc}
x_{1} & w_{1,2}^{D} & w_{1,3}^{D} & \cdots & w_{1, n}^{D} \\
x_{2} & & w_{2,3}^{D} & \cdots & w_{2, n}^{D} \\
\vdots & & & \vdots & \vdots \\
x_{n-1} & & & & w_{n-1, n}^{D}
\end{array}\right]=\left[\begin{array}{ll}
X & W^{D}
\end{array}\right],
$$

where,

$$
\begin{aligned}
& X=\left[\begin{array}{c}
x_{1} \\
x_{2} \\
\vdots \\
x_{n-1}
\end{array}\right] \text { is the object vector } \\
& \text { - } W^{D}=\left[\begin{array}{cccc}
w_{1,2}^{D} & w_{1,3}^{D} & \cdots & w_{1, n}^{D} \\
& w_{2,3}^{D} & \cdots & w_{2, n}^{D} \\
& & \vdots & \vdots \\
& & w_{n-1, n}^{D}
\end{array}\right] \text { is the feature value matrix. }
\end{aligned}
$$

Definitions 13 and 14 greatly improve the utilization of computer memory. For a universe with $n$ objects, matrix $W^{D}$ has $2 n^{2}$ binary bits by Definition 12 while has only $n(n-1)$ bits by Definition 14 . Dominance feature matrix directly indicates the dominance relations between any two objects in the universe. This means that after new objects and attributes are added into an information system, we have known the dominance relations between objects with respect to their old attributes. Thus we can update approximations by new attributes without considering old ones again.

Lemma 1. Let $S=(U, C \cup\{d\}, V, f)$ be an ordered information system with $n$ objects in $U$ and $m$ attributes in $C$. When $l$ new objects which comprise an additional universe $U^{+}$, and $q$ new attributes which comprise an additional attribute set $C^{+}$are simultaneously added to $S$. Let $U^{\prime}=U \cup U^{+}$be the updated universe, while $C^{\prime}=C \cup C^{+}$be the updated attribute set. Then the updated dominance feature flag can be obtained by the following rules. 
(1) Let $w_{i, j}^{D \prime}$ be the updated dominance flag after new objects are processed. We have:

$$
w_{i, j}^{D^{\prime}}=\left\{\begin{array}{ll}
00, & f\left(x_{i}, a_{k}\right) \otimes f\left(x_{j}, a_{k}\right) \\
01, & f\left(x_{i}, a_{k}\right)>f\left(x_{j}, a_{k}\right) \\
10, & f\left(x_{i}, a_{k}\right) \prec f\left(x_{j}, a_{k}\right) \\
11, & f\left(x_{i}, a_{k}\right)=f\left(x_{j}, a_{k}\right)
\end{array},\right.
$$

where, $\forall x_{i} \in U, i \in\{1,2, \cdots, n\}, \forall x_{j} \in U^{+}, j \in\{n+1, n+2, \cdots, n+l\}, \forall a_{k} \in C, k \in\{1,2, \cdots, m\} ;$

(2) Let $w_{i, j}^{D \prime \prime}$ be the updated dominance flag after new attributes are processed. We have:

$$
w_{i, j}^{D^{\prime \prime}}=\left\{\begin{array}{l}
00, \quad f\left(x_{i}, a_{k}\right) \otimes f\left(x_{j}, a_{k}\right) \\
w_{i, j}^{D^{\prime}} \& \& 01, \quad f\left(x_{i}, a_{k}\right)>f\left(x_{j}, a_{k}\right) \\
w_{i, j}^{D^{\prime}} \& \& 10, \quad f\left(x_{i}, a_{k}\right)<f\left(x_{j}, a_{k}\right) \\
w_{i, j}^{D^{\prime},} \quad f\left(x_{i}, a_{k}\right)=f\left(x_{j}, a_{k}\right)
\end{array},\right.
$$

where, operator "\&\&” denotes performing a bitwise AND, $\forall x_{i}, x_{j} \in U^{\prime}, i, j \in\{1,2, \cdots, n+l\}, \forall a_{k} \in C^{+}$, $k \in\{m+1, m+2, \cdots, m+q\}$.

Proof. (1) It follows directly from Definition 10.

(2) For $x_{i}, x_{j} \in U^{\prime}, \forall a_{k} \in C^{+}$, if $f\left(x_{i}, a_{k}\right) \otimes f\left(x_{j}, a_{k}\right)$, then $x_{i}$ must be dominance independent to $x_{j}$ with respect to $C^{\prime}$. Thus $w_{i, j}^{D \prime \prime}=00$; Conversely, if $f\left(x_{i}, a_{k}\right)=f\left(x_{j}, a_{k}\right)$, then the updated dominance relation between $x_{i}$ and $x_{j}$ is decided by their old relations with respect to $C$. Thus $w_{i, j}^{D^{\prime \prime}}=w_{i, j}^{D^{\prime}}$; If $f\left(x_{i}, a_{k}\right)>f\left(x_{j}, a_{k}\right)$, then $w_{i, j}^{D \prime \prime}=01$ when $w_{i, j}^{D^{\prime}}=01$ or $w_{i, j}^{D^{\prime}}=11$, otherwise $w_{i, j}^{D^{\prime \prime}}=00$ when $w_{i, j}^{D^{\prime}}=00$ or $w_{i, j}^{D^{\prime}}=10$. Thus $w_{i, j}^{D^{\prime \prime}}=w_{i, j}^{D^{\prime}}$ \&\& 01; Analogously, $w_{i, j}^{D \prime \prime}=w_{i, j}^{D^{\prime}} \& \& 10$.

Following is an example of a complete process that updates approximations of DRSA incrementally when new objects and attributes are added simultaneously.

Example 4. Table 3 illustrates an ordered information system originates from Table 1 with simultaneously added objects $x_{9}, x_{10}$ and attributes $a_{3}, a_{4}$. Let $U^{\prime}=U \cup\left\{x_{9}, x_{10}\right\}$ be the updated universe, and $C^{\prime}=C \cup\left\{a_{3}, a_{4}\right\}$ be the updated set of condition attributes.

From Definition 14, the dominance feature matrix of Table 1 is :

$$
M^{D}=\left[\begin{array}{c}
V_{1}^{D} \\
V_{2}^{D} \\
V_{3}^{D} \\
V_{4}^{D} \\
V_{5}^{D} \\
V_{6}^{D} \\
V_{7}^{D}
\end{array}\right]=\left[\begin{array}{llllllll}
x_{1} & 00 & 10 & 01 & 10 & 10 & 10 & 10 \\
x_{2} & & 10 & 00 & 10 & 10 & 10 & 10 \\
x_{3} & & & 01 & 10 & 10 & 10 & 10 \\
x_{4} & & & & 10 & 10 & 10 & 10 \\
x_{5} & & & & & 00 & 01 & 10 \\
x_{6} & & & & & & 01 & 10 \\
x_{7} & & & & & & & 10
\end{array}\right] .
$$

According to Lemma 1, firstly the new objects $x_{9}$ and $x_{10}$ are processed, we have:

$$
\begin{gathered}
w_{1,9}^{D}{ }^{\prime}=10, w_{2,9}^{D}{ }^{\prime}=10, w_{3,9}^{D}{ }^{\prime}=10, w_{4,9}^{D}{ }^{\prime}=10, w_{5,9}^{D}{ }^{\prime}=00, w_{6,9}^{D}{ }^{\prime}=11, w_{7,9}^{D}{ }^{\prime}=10, w_{8,9}^{D}{ }^{\prime}=01 ; \\
w_{1,10}^{D}{ }^{\prime}=00, w_{2,10}^{D}{ }^{\prime}=10, w_{3,10}^{D}{ }^{\prime}=00, w_{4,10}^{D}{ }^{\prime}=00, w_{5,10}^{D}{ }^{\prime}=01, w_{6,10}^{D}{ }^{\prime}=01, w_{7,10}^{D}{ }^{\prime}=01, w_{8,10}^{D}{ }^{\prime}=01, w_{9,10}^{D}{ }^{\prime}=01 .
\end{gathered}
$$


Table 3: An ordered information system with simultaneously added objects and attributes.

\begin{tabular}{llllll}
\hline$U^{\prime}$ & $a_{1}$ & $a_{2}$ & $a_{3}$ & $a_{4}$ & $d$ \\
\hline$x_{1}$ & 40 & 65 & 50 & 70 & 2 \\
$x_{2}$ & 55 & 40 & 80 & 55 & 1 \\
$x_{3}$ & 60 & 65 & 60 & 65 & 1 \\
$x_{4}$ & 40 & 50 & 55 & 45 & 1 \\
$x_{5}$ & 70 & 80 & 65 & 45 & 2 \\
$x_{6}$ & 80 & 70 & 40 & 55 & 3 \\
$x_{7}$ & 70 & 70 & 50 & 70 & 3 \\
$x_{8}$ & 80 & 80 & 80 & 60 & 3 \\
$x_{9}$ & 80 & 70 & 60 & 75 & 3 \\
$x_{10}$ & 65 & 45 & 80 & 60 & 2 \\
\hline
\end{tabular}

Let $V_{i}^{D^{\prime}}$ and $M^{D^{\prime}}$ be the updated dominance feature vector and matrix respectively after objects $x_{9}$ and $x_{10}$ are processed. We have:

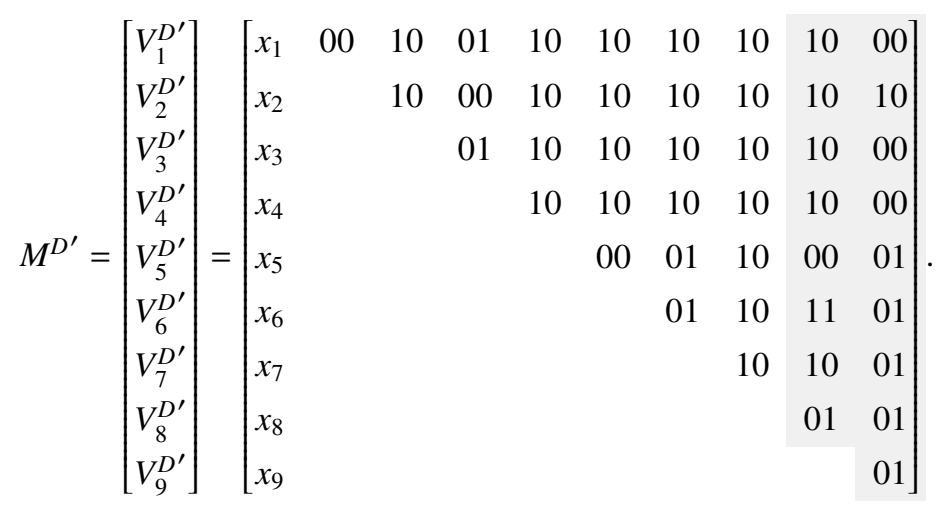

Compared with $M^{D}, M^{D^{\prime}}$ is in fact a matrix with newly added columns with respect to the new dominance feature flags.

Then we process new attributes $a_{3}$ and $a_{4}$. For all objects, we have known their dominance relations with respect to $a_{1}$ and $a_{2}$ by $w_{i, j}^{D^{\prime}}$. Thus according to Lemma 1 , we can incrementally update $w_{i, j}^{D \prime \prime}$ only by $a_{3}$ and $a_{4}$. So we have:

$$
\begin{aligned}
& w_{1,3}^{D \prime \prime}=00, w_{1,4}^{D \prime \prime}=00, w_{1,5}^{D \prime \prime}=00, w_{1,6}^{D \prime \prime}=00, w_{1,8}^{D \prime}=00 ; \\
& w_{2,3}^{D} "=00, w_{2,5}^{D} "=00, w_{2,6}^{D} "=00, w_{2,7}^{D} "=00, w_{2,9}^{D} "=00 ; \\
& w_{3,5}^{D \prime \prime}=00, w_{3,6}^{D} "=00, w_{3,7}^{D \prime \prime}=00, w_{3,8}^{D}{ }^{\prime \prime}=00 \\
& w_{4,6}^{D} "=00, w_{4,7}^{D} "=00 \text {; } \\
& w_{5,7}^{D} "=00, w_{5,10}^{D} "=00 \\
& w_{6,7}^{D \prime \prime}=00, w_{6,9}^{D \prime \prime}=10, w_{6,10}^{D}{ }^{\prime \prime}=00 \text {; } \\
& w_{7,8}^{D \prime \prime}=00, w_{7,10}^{D}{ }^{\prime \prime}=00 \\
& w_{8,9}^{D \prime}=00 ; \\
& w_{9,10}^{D}{ }^{\prime \prime}=00 .
\end{aligned}
$$

Let $V_{i}^{D^{\prime \prime}}$ and $M^{D^{\prime \prime}}$ be the updated dominance feature vector and matrix respectively after attributes $a_{3}$ and $a_{4}$ are processed. We have: 


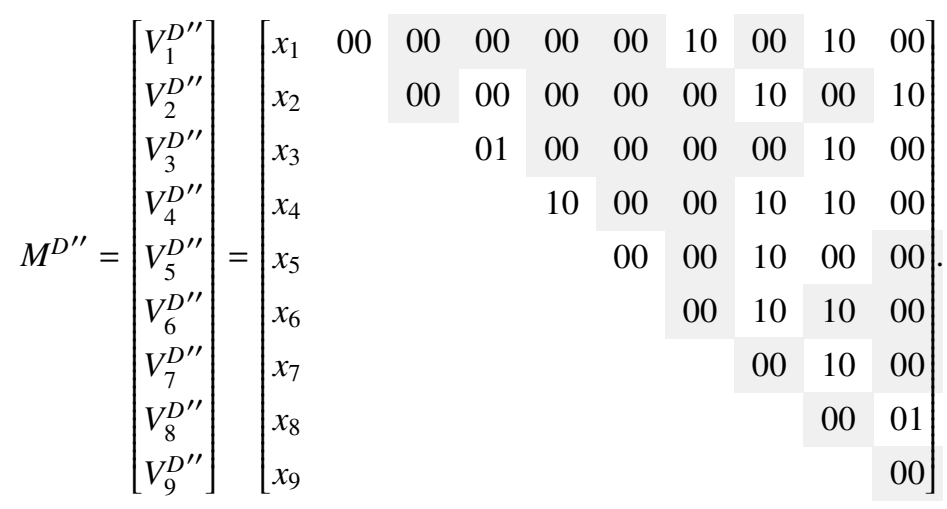

According to Definition 9, we have:

$$
\begin{array}{ll}
l_{P}\left(x_{1}\right)=2, & u_{P}\left(x_{1}\right)=2 \\
l_{P}\left(x_{2}\right)=1, & u_{P}\left(x_{2}\right)=1 \\
l_{P}\left(x_{3}\right)=1, & u_{P}\left(x_{3}\right)=1 \\
l_{P}\left(x_{4}\right)=1, & u_{P}\left(x_{4}\right)=1 \\
l_{P}\left(x_{5}\right)=2, & u_{P}\left(x_{5}\right)=2 \\
l_{P}\left(x_{6}\right)=2, & u_{P}\left(x_{6}\right)=3 \\
l_{P}\left(x_{7}\right)=2, & u_{P}\left(x_{7}\right)=3 \\
l_{P}\left(x_{8}\right)=3, & u_{P}\left(x_{8}\right)=3 \\
l_{P}\left(x_{9}\right)=2, & u_{P}\left(x_{9}\right)=3 \\
l_{P}\left(x_{10}\right)=3, & u_{P}\left(x_{10}\right)=3
\end{array}
$$

Finally according to Definition 8, we obtain approximations of this information system as follows:

$$
\begin{gathered}
\underline{P}\left(C l_{1}^{\geq}\right)=U^{\prime}, \bar{P}\left(C l_{1}^{\geq}\right)=U^{\prime}, \underline{P}\left(C l_{1}^{\leq}\right)=\left\{x_{2}, x_{3}, x_{4}\right\}, \bar{P}\left(C l_{1}^{\leq}\right)=\left\{x_{2}, x_{3}, x_{4}\right\} ; \\
\underline{P}\left(C l_{2}^{\geq}\right)=\left\{x_{1}, x_{5}, x_{6}, x_{7}, x_{8}, x_{9}, x_{10}\right\}, \bar{P}\left(C l_{2}^{\geq}\right)=\left\{x_{1}, x_{5}, x_{6}, x_{7}, x_{8}, x_{9}, x_{10}\right\}, \\
\underline{P}\left(C l_{2}^{\leq}\right)=\left\{x_{1}, x_{2}, x_{3}, x_{4}, x_{5}\right\}, \bar{P}\left(C l_{2}^{\leq}\right)=\left\{x_{1}, x_{2}, x_{3}, x_{4}, x_{5}, x_{6}, x_{7}, x_{9}\right\} ; \\
\underline{P}\left(C l_{3}^{l}\right)=\left\{x_{8}, x_{10}\right\}, \bar{P}\left(C l_{3}^{\geq}\right)=\left\{x_{6}, x_{7}, x_{8}, x_{9}, x_{10}\right\}, \underline{P}\left(C l_{3}^{\leq}\right)=U^{\prime}, \bar{P}\left(C l_{3}^{\leq}\right)=U^{\prime} .
\end{gathered}
$$

Although we reduce the memory consumption by Definitions 13 and 14, the vector $V_{i}^{D}$ and the matrix $W^{D}$ in Example 4 are still large for massive data. So they need to be further improved. From Definition 10, we find that there is always 0 in $w_{i, j}^{D}$ except for the value 11 , so there should be 0 in vector $V_{i}^{D}$ and matrix $W^{D}$. Furthermore, it can be inferred that the number of 11 is much fewer than that of other three values in $V_{i}^{D}$ and $W^{D}$, because real data always carries a number of attributes which often lead to few equal objects. In Table 1, if we only consider attribute $a_{1}$, then $x_{1}=x_{4}, x_{5}=x_{7}, x_{6}=x_{8}$. However, in view of attribute $a_{1}$ and $a_{2}$ altogether, there are not any objects are equal again. The more the attributes, the fewer the equal objects, that is the fewer 11 . Fewer 11 means more $0 \sin V_{i}^{D}$ and $W^{D}$, so $V_{i}^{D}$ can be viewed as a sparse vector while $W^{D}$ can be viewed as a sparse matrix. If we only record all $1 \mathrm{~s}$ in $V_{i}^{D}$ and $W^{D}$, then the memory consumption can be reduced. Furthermore, from Definition 9 , we know that for an object $x$, if its P-generalized decision $l_{P}(x)$ needs to be updated, two conditions must be satisfied. First, there exists at least one object which is dominating $x$ in the universe. Second, the decision attribute values of those objects dominating $x$ are less than current $l_{P}(x)$. That is, if any objects are dominating $x$, and their decision attribute values are larger than or equal to current $l_{P}(x)$, then these objects cannot affect $l_{P}(x)$. Therefore, the dominance feature flags of $x$ and these objects do not need to be recorded. Analogously, for those objects which are dominated by $x$ and whose decision attribute values are less than or equal to current $u_{P}(x)$, they cannot affect $u_{P}(x)$. Therefore, the dominance feature flags of $x$ and these objects also do not need to be recorded.

By all of the above strategies, the memory consumption may be fairly reduced. Here we use the following example to show the effectiveness of these strategies. 
Example 5. (Continuation of Example 4)

We still take object $x_{1}$ for example. According to Definition 13, before new objects and attributes are added, the dominance feature vector of $x_{1}$ should be recorded as follows :

$$
V_{1}^{D}=\left(x_{1} 00100110101010\right) .
$$

We initialize $l_{P}\left(x_{1}\right)=2$ and $u_{P}\left(x_{1}\right)=2$, for the decision attribute value of $x_{1}$ is 2 . In the universe $U$, although objects $x_{3}, x_{5}, x_{6}, x_{7}, x_{8}$ are all dominating $x_{1}$, only the decision attribute value of $x_{3}$ is less than $l_{P}\left(x_{1}\right)$. Thus only the dominance feature flag of $x_{1}$ and $x_{3}$ needs to be recorded. Analogously, although object $x_{4}$ is dominated by $x_{1}$, the decision attribute value of $x_{4}$ is less than that of $x_{1}$, which means $x_{4}$ cannot affect $u_{P}\left(x_{1}\right)$. Thus the dominance feature flag of $x_{1}$ and $x_{4}$ does not need to be recorded. Then vector $V_{1}^{D}$ can be recorded as follows :

$$
V_{1}^{D}=\left(\begin{array}{lll}
x_{1} & 1
\end{array}\right) .
$$

Here the line beside 1 is in fact a 0 , which also does not need to be recorded. Thus "I_" denotes 10 and "_l" denotes 01 .

So we improve matrix $M^{D}$ in Example 4 as follows:

$$
M^{D}=\left[\begin{array}{c}
V_{1}^{D} \\
V_{2}^{D} \\
V_{3}^{D} \\
V_{4}^{D} \\
V_{5}^{D} \\
V_{6}^{D} \\
V_{7}^{D}
\end{array}\right]=\left[\begin{array}{lll}
x_{1} & 1- & \\
x_{2} & & \\
x_{3} & & \\
x_{4} & & - \\
x_{5} & & \\
x_{6} & & \\
x_{7} & &
\end{array}\right]
$$

And the improved matrices $M^{D^{\prime}}$ and $M^{D^{\prime \prime}}$ are as follows:

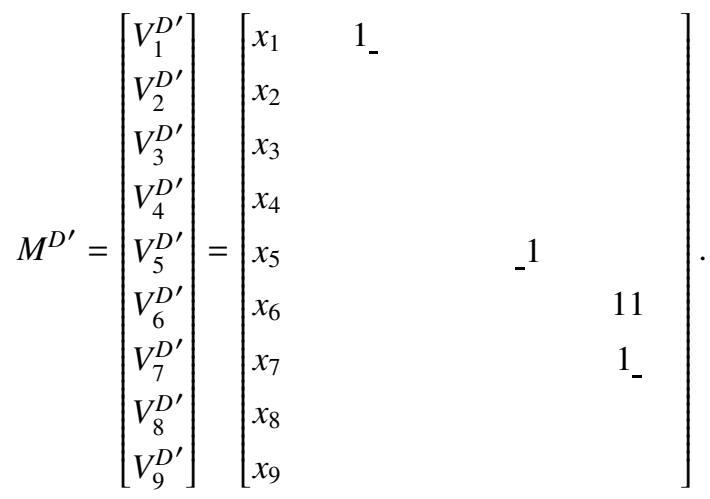

$$
\begin{aligned}
& M^{D^{\prime \prime}}=\left[\begin{array}{c}
V_{1}^{D^{\prime \prime}} \\
V_{2}^{D^{\prime \prime}} \\
V_{3}^{D^{\prime \prime}} \\
V_{4}^{D^{\prime \prime}} \\
V_{5}^{D^{\prime \prime}} \\
V_{6}^{D^{\prime \prime}} \\
V_{7}^{D^{\prime \prime}} \\
V_{8}^{D^{\prime \prime}} \\
V_{9}^{D^{\prime \prime}}
\end{array}\right]=\left[\begin{array}{ll}
x_{1} & \\
x_{2} & \\
x_{3} & \\
x_{4} & \\
x_{5} & 1 \\
x_{6} & 1 \\
x_{7} & \\
x_{8} & \\
x_{9} &
\end{array}\right] .
\end{aligned}
$$


By the storage scheme in Example 5, the memory consumption is greatly reduced. Significantly, we notice that the memory consumption of matrix $M^{D^{\prime \prime}}$ is even less than that of matrix $M^{D^{\prime}}$, because after new attributes are processed, some $01 \mathrm{~s}$ and $10 \mathrm{~s}$ may change to 00s. In real data, for two objects $x_{i}$ and $x_{j}$ with a lot of attributes, it is common that $x_{i}$ may be better than $x_{j}$ with respect to some attributes, while be worse than $x_{j}$ with respect to other attributes. That is, the probability that any two objects are dominance independent to each other is high, and with the addition of more attributes, the probability increases. From Fig. 1, we can clearly see the change of $w_{i, j}^{D}$ after new attributes are processed. The number of 0 has a great potential to increase.

$$
w_{i, j}^{D}=\left\{\begin{aligned}
00 & \rightarrow 00 \\
01 & \rightarrow\left\{\begin{array}{l}
01 \\
00
\end{array}\right. \\
10 & \rightarrow\left\{\begin{array}{l}
10 \\
00
\end{array}\right. \\
11 & \rightarrow\left\{\begin{array}{l}
11 \\
10 \\
01
\end{array}\right.
\end{aligned}\right.
$$

Figure 1: The change of $w_{i, j}^{D}$ when new attributes are processed.

Therefore, the storage scheme in Example 5 is very effective to reduce memory consumption. In the worst case, all the values in matrix $M^{D}$ are $11 \mathrm{~s}$, which means all objects are equal, and the P-generalized decision values of any object are affected by the decision attribute values of other objects. Then $M^{D}$ has to consume the memory as much as it does in Example 4. However, such case is almost impossible for real data, especially for real massive data.

\section{The algorithm for updating approximations in DRSA when objects and attributes increase simultaneously}

According to the definitions, propositions, lemma and examples in Sections 2 and 3, we design the algorithm in this section to update approximations in DRSA.

We integrate the simplified strategy with the incremental learning strategy proposed in Section 3, then design the Incremental Simplified Algorithm (ISA) as shown in Algorithm 1. This algorithm avoids repeated comparisons between old attributes and neglects unnecessary steps for getting P-generalized decision so as to update approximations efficiently. For an information system with $n$ objects, $m$ attributes and $r$ decision values, when $l$ new objects and $q$ new attributes are added simultaneously, in the best case, the time complexity of Non-Incremental Algorithm (NIA) in [22] is $O(r(n+l)(1+(n+l)(m+q)))$, while in the worst case, the time complexity of ISA is only $O\left(m \cdot \sum_{k=1}^{l}(n+k)(n+k-1)+q \cdot \sum_{j=1}^{n+l-1} k(n+l-k)+r\right)$.

Since two different storage schemes are exemplified in Section 3, there are two implementations for ISA. We call the first one the Incremental Simplified Algorithm based on the Dominance Feature Matrix for updating approximations in DRSA (ISA-DFM). ISA-DFM employs the storage scheme in Example 4, so the dominance feature matrix is relatively memory-consuming. The second implementation for ISA is called the Incremental Simplified Algorithm based on Improved Dominance Feature Matrix for updating approximations in DRSA (ISA-IDFM), which employs the storage scheme in Example 5. Although these two implementations are all based on Algorithm 1, they are rather different. ISA-DFM can directly get the dominance feature flag $w_{i, j}^{D}$ from the feature value matrix $W^{D}$, while ISAIDFM needs to decide $w_{i, j}^{D}$ by the position of 1 in $W^{D}$. In programming, we can define a higher bit and a lower bit to denote $w_{i, j}^{D}$ of two objects $x_{i}$ and $x_{j}$. Both the higher and lower bits exist in $W^{D}$ means $w_{i, j}^{D}=11$, namely $x_{i}=x_{j}$, while only the higher or lower bit exists means $w_{i, j}^{D}=10$ or $w_{i, j}^{D}=01$, namely $x_{i} \prec x_{j}$ or $x_{i}>x_{j}$ respectively, and if none of the two bits exists, then $x_{i}$ is independent to $x_{j}$. 


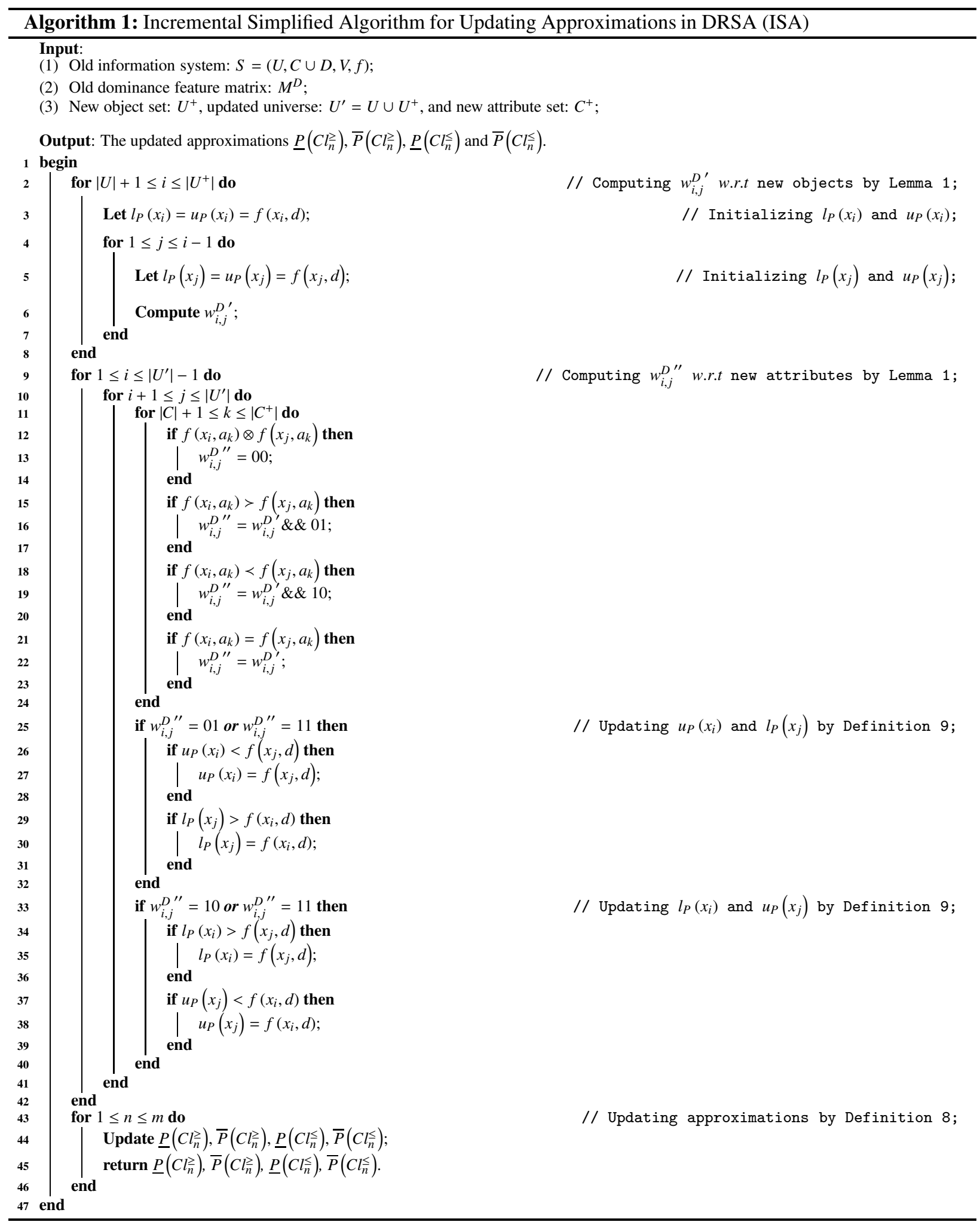




\section{Experimental evaluations}

For evaluating the performance of our proposed incremental simplified algorithm, in this section we conduct two groups of experiments based on the data sets shown in Table 4. Most of the data sets come from the machine learning data repository, University of California at Irvine (UCI) [3], while the others are artificial. In data set Mice Protein Expression, we delete some objects and attributes with missing values.

Table 4: A description of data sets.

\begin{tabular}{llccc}
\hline Data sets & Abbreviation & Objects & Attributes & Classes \\
\hline Wine & Wine & 178 & 13 & 3 \\
Sonar & Sonar & 208 & 60 & 2 \\
Leaf & Leaf & 340 & 15 & 30 \\
Ionosphere & Ionosphere & 351 & 34 & 2 \\
Libras Movement & Libras & 360 & 90 & 15 \\
Robot Execution Failures & Robot & 463 & 90 & 16 \\
Mice Protein Expression & Mice & 1,077 & 68 & 8 \\
Artificial Data 1 & AD1 & 5,265 & 34 & 2 \\
Artificial Data 2 & AD2 & 49,772 & 34 & 2 \\
\hline
\end{tabular}

In order to test the algorithms by different data sizes, for each data set in Table 4 , we take $50 \%$ data from it to comprise a basic data block, and make this basic block increase in the proportion of $10 \%, 20 \%, 30 \%, 40 \%$, $50 \%$, respectively. Thus we get five different-sized data blocks in the proportion of $60 \%, 70 \%, 80 \%, 90 \%, 100 \%$, respectively of this data set. In each group of experiments, for each data set, five separate experiments are successively conducted based on the five different-sized data blocks of this data set. Each separate experiment employs only one data block. All algorithms are implemented by $\mathrm{C}++$ programming language in a Visual Studio 2013 Ultimate edition, and all experiments are conducted on a computer with $4.0 \mathrm{GHz}$ CPU, 16GB memory and 64-bit Windows 7 Ultimate Sp1 system.

\subsection{A comparison of non-incremental and incremental simplified algorithms when objects and attributes increase simultaneously}

In this group of experiments, we compare the efficiency of our proposed incremental simplified algorithm (ISA) with the non-incremental one (NIA) in [22]. We also want to check the efficiency difference between two implementations ISA-DFM and ISA-IDFM of ISA, so NIA, ISA-DFM and ISA-IDFM are compared altogether. Both the non-incremental algorithm and the incremental simplified algorithm are tested by the five data blocks. Computational time is shown in Table 5 and corresponding curves are illustrated in Fig. 2.

The $x$-coordinates of all sub-figures (a)-(i) in Fig. 2 denote sizes of data blocks, while the $y$-coordinates present the computational time. The curves in different styles in all sub-figures show the trends of computational time of different algorithms. We can easily see that with the increasing of data size in all data sets, computational time of NIA, ISA-DFM and ISA-IDFM also increases, but ISA-DFM and ISA-IDFM are always faster than NIA, especially when the data size gets larger and larger, such a speed advantage is more and more obvious. On the other side, the efficiency differences between ISA-DFM and ISA-IDFM are fairly little in all data sets. What's more, we notice that ISA-DFM does not get the computational time when the data size is $90 \%$ or $100 \%$ in data set AD2, because the computer memory has run out. But benefits from the improved reduced storage scheme in Example 5, ISA-IDFM can still work at that time. Therefore, for our proposed incremental simplified approach, ISA-IDFM is a better choice than ISA-DFM.

To show the efficiency advantage of our proposed algorithm more clearly, we also compute the speed-up ratio that ISA-IDFM relates to NIA. Since we have verified that ISA-IDFM is the better choice, here we compare it with NIA without considering ISA-DFM. The results are shown in Fig. 3(a).

From Fig. 3(a), we notice that for all data sets, ISA-IDFM is nearly 10 times or more faster than NIA. Especially in some data sets such as Robot, Libras, Mice and Leaf, ISA-IDFM is even tens of times faster than NIA. The results show once again the better performance of our proposed incremental simplified algorithm in efficiency. We also find 

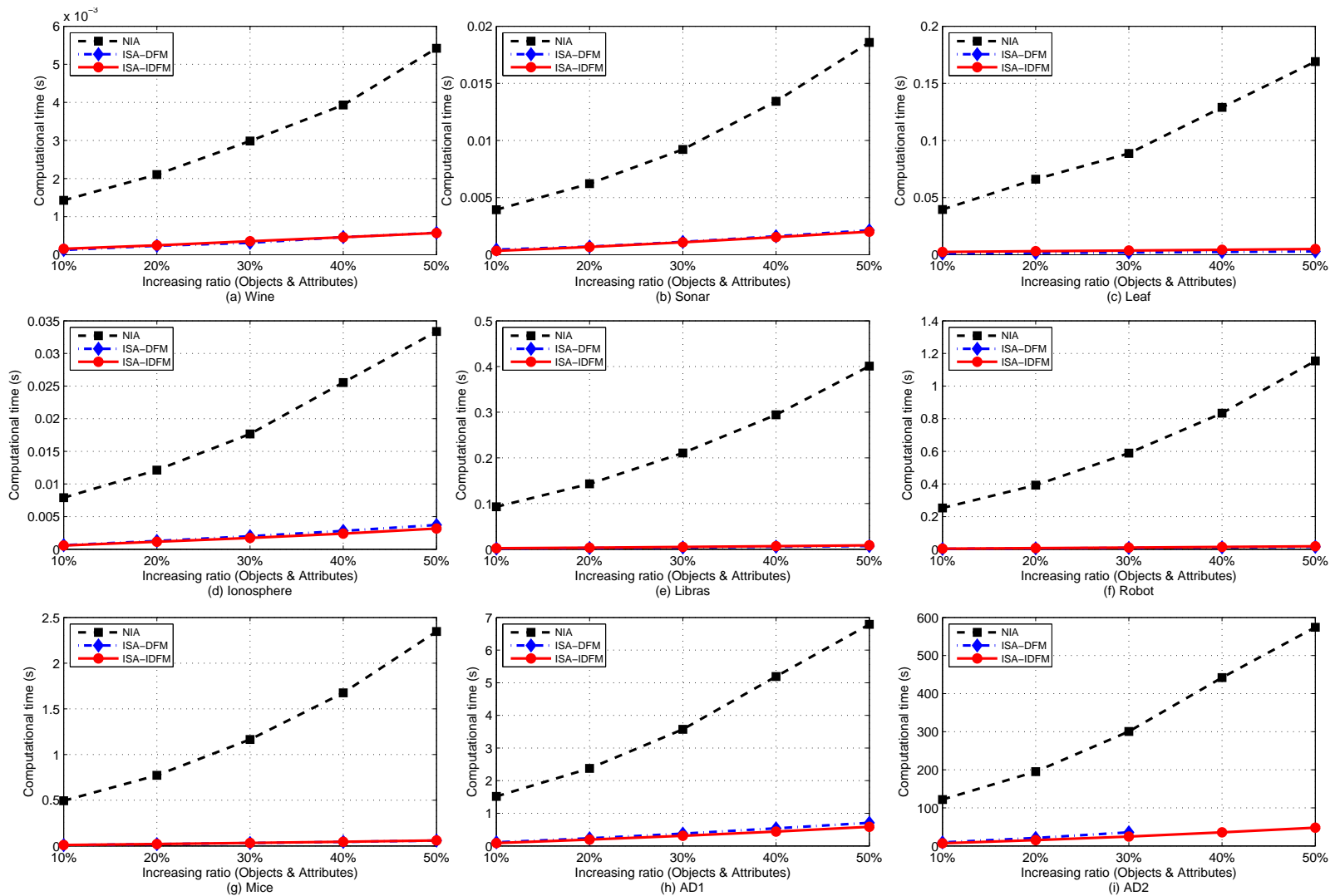

Figure 2: Comparisons of computational time between non-incremental algorithm and incremental simplified algorithm when objects and attributes increase simultaneously. 
Table 5: Comparisons of computational time between non-incremental algorithm and incremental simplified algorithm when objects and attributes increase simultaneously.

\begin{tabular}{|c|c|c|c|c|c|c|c|c|c|c|c|c|c|c|c|}
\hline \multirow[b]{2}{*}{ Data size } & \multicolumn{3}{|c|}{ Wine } & \multicolumn{3}{|c|}{ Sonar } & \multicolumn{3}{|c|}{ Leaf } & \multicolumn{3}{|c|}{ Ionosphere } & \multicolumn{3}{|c|}{ Libras } \\
\hline & NIA & DFM & IDFM & NIA & DFM & IDFM & NIA & DFM & IDFM & NIA & DFM & IDFM & NIA & DFM & IDFM \\
\hline $60 \%$ & 0.0014 & 0.0001 & 0.0002 & 0.0039 & 0.0005 & 0.0003 & 0.0396 & 0.0011 & 0.0024 & 0.0079 & 0.0006 & 0.0006 & 0.0929 & 0.0014 & 0.0021 \\
\hline $70 \%$ & 0.0021 & 0.0002 & 0.0002 & 0.0062 & 0.0007 & 0.0007 & 0.0661 & 0.0016 & 0.0031 & 0.0121 & 0.0013 & 0.0011 & 0.1432 & 0.0026 & 0.0035 \\
\hline $80 \%$ & 0.0029 & 0.0003 & 0.0004 & 0.0092 & 0.0011 & 0.0011 & 0.0886 & 0.002 & 0.0037 & 0.0177 & 0.002 & 0.0017 & 0.2107 & 0.004 & 0.005 \\
\hline $90 \%$ & 0.0039 & 0.0005 & 0.0005 & 0.0134 & 0.0016 & 0.0015 & 0.129 & 0.0024 & 0.0043 & 0.0255 & 0.0028 & 0.0024 & 0.2942 & 0.0055 & 0.0067 \\
\hline \multirow[t]{2}{*}{$100 \%$} & 0.0054 & 0.0006 & 0.0006 & 0.0186 & 0.0021 & 0.002 & 0.1691 & 0.0029 & 0.005 & 0.0334 & 0.0037 & 0.0032 & 0.4008 & 0.0071 & 0.0086 \\
\hline & \multicolumn{3}{|c|}{ Robot } & \multicolumn{3}{|c|}{ Mice } & \multicolumn{3}{|c|}{ AD1 } & \multicolumn{3}{|c|}{ AD2 } & & & \\
\hline Data size & NIA & DFM & IDFM & NIA & DFM & IDFM & NIA & DFM & IDFM & NIA & DFM & IDFM & & & \\
\hline $60 \%$ & 0.253 & 0.0025 & 0.0039 & 0.494 & 0.0089 & 0.0099 & 1.5165 & 0.1116 & 0.0889 & 121.714 & 9.2419 & 6.9834 & & & \\
\hline $70 \%$ & 0.3931 & 0.0053 & 0.0069 & 0.7726 & 0.0192 & 0.0203 & 2.3754 & 0.2353 & 0.1924 & 195.11 & 20.8289 & 15.1909 & & & \\
\hline $80 \%$ & 0.589 & 0.0083 & 0.0103 & 1.165 & 0.0311 & 0.0322 & 3.5733 & 0.3756 & 0.3089 & 300.573 & 35.6349 & 24.7012 & & & \\
\hline $90 \%$ & 0.8339 & 0.0114 & 0.014 & 1.6751 & 0.0439 & 0.0453 & 5.1924 & 0.5381 & 0.4408 & 442.175 & - & 35.6085 & & & \\
\hline $100 \%$ & 1.1546 & 0.0152 & 0.0182 & 2.3468 & 0.0588 & 0.0604 & 6.7886 & 0.7089 & 0.5846 & 574.285 & - & 47.7892 & & & \\
\hline
\end{tabular}

that the speed-up ratios in data sets Wine, Sonar, Ionosphere, AD1 and AD2 are much lower than those in Leaf, Mice, Libras and Robot, while the classes in the latter four are much more than those in the former five. This suggests that the efficiency of our proposed algorithm depends much on the number of classes in data. The more the classes, the higher the algorithm efficiency. For example, the sizes of AD1 and AD2 are all much larger than those of Leaf, Mice, Libras and Robot, however the speed-up ratios in AD1 and AD2 are lower. The above cases illustrates that our proposed incremental simplified algorithm is especially more efficient for processing multi-label data.

\subsection{A comparison of the integration of two single-dimensional incremental algorithms and incremental simplified algorithm when objects and attributes increase simultaneously}

For individual increasing of objects or attributes in DRSA, Li et al. proposed two corresponding single-dimensional incremental algorithms [22, 23]. For convenience, we call these two algorithms IAUAO (Incremental Algorithm for Updating Approximations of DRSA when a new Object enters an information system) in [22] and IAUAA (Incremental Algorithm for Updating Approximations of DRSA when some new Attributes are added into the information system) in [23] respectively. When objects and attributes increase simultaneously, if we integrate these two singledimensional algorithms to successively deal with new objects and new attributes, the approximations can also be updated. To verify the effectiveness of our proposed incremental simplified algorithms for specially dealing with the simultaneously added objects and attributes, we integrate IAUAO with IAUAA, then compare the efficiencies of the integrated algorithm IAUAO+IAUAA with our proposed incremental simplified algorithm ISA-IDFM. We employ some data sets with markedly different numbers of classes in this group of experiments. The computational time is shown in Table 6 and the corresponding curves are illustrated in Fig. 4.

From Table 6 and Fig. 4, it is obvious that our proposed algorithm ISA-IDFM is also much faster than the integration of two single-dimensional algorithms IAUAO and IAUAA, notably when the data size gets larger. From the speed-up ratio curves in Fig. 3(b), we notice that the speed-up ratio keeps relative stable in the data sets with only a few classes, such as Wine, Sonar and Ionosphere, while monotonously increases in the data sets with much more classes, such as Leaf and Libras. These phenomena show that IAUAO+IAUAA may be delayed more by a dozen or more classes. In addition, IAUAO is based on traditional definition of P-generalized decision, that is, IAUAO still needs to compute the dominating and dominated sets as well as the decision classes. So in real-life problems, when massive objects and attributes enter the information system, the integrated IAUAO+IAUAA will be very timeconsuming. All the facts above mean that our proposed incremental simplified algorithm is efficient for specially processing simultaneously added objects and attributes in DRSA. 

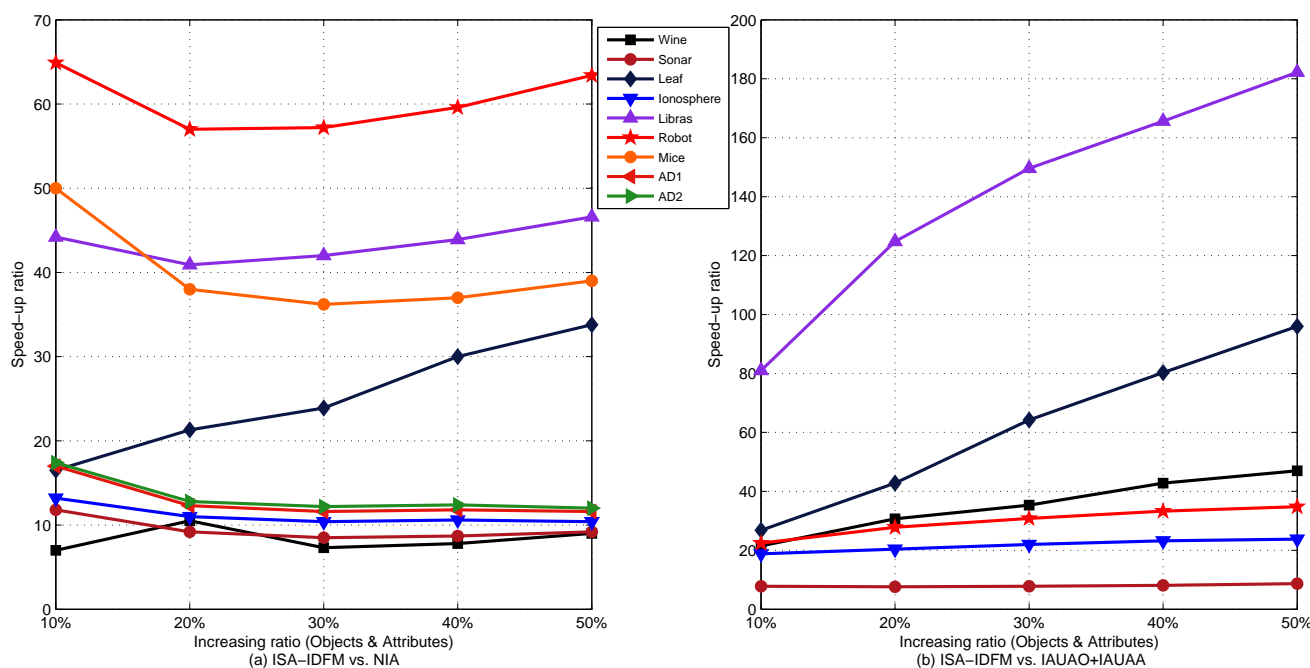

Figure 3: The speed-up ratios that incremental simplified algorithm relates to non-incremental algorithm and integrated algorithms.
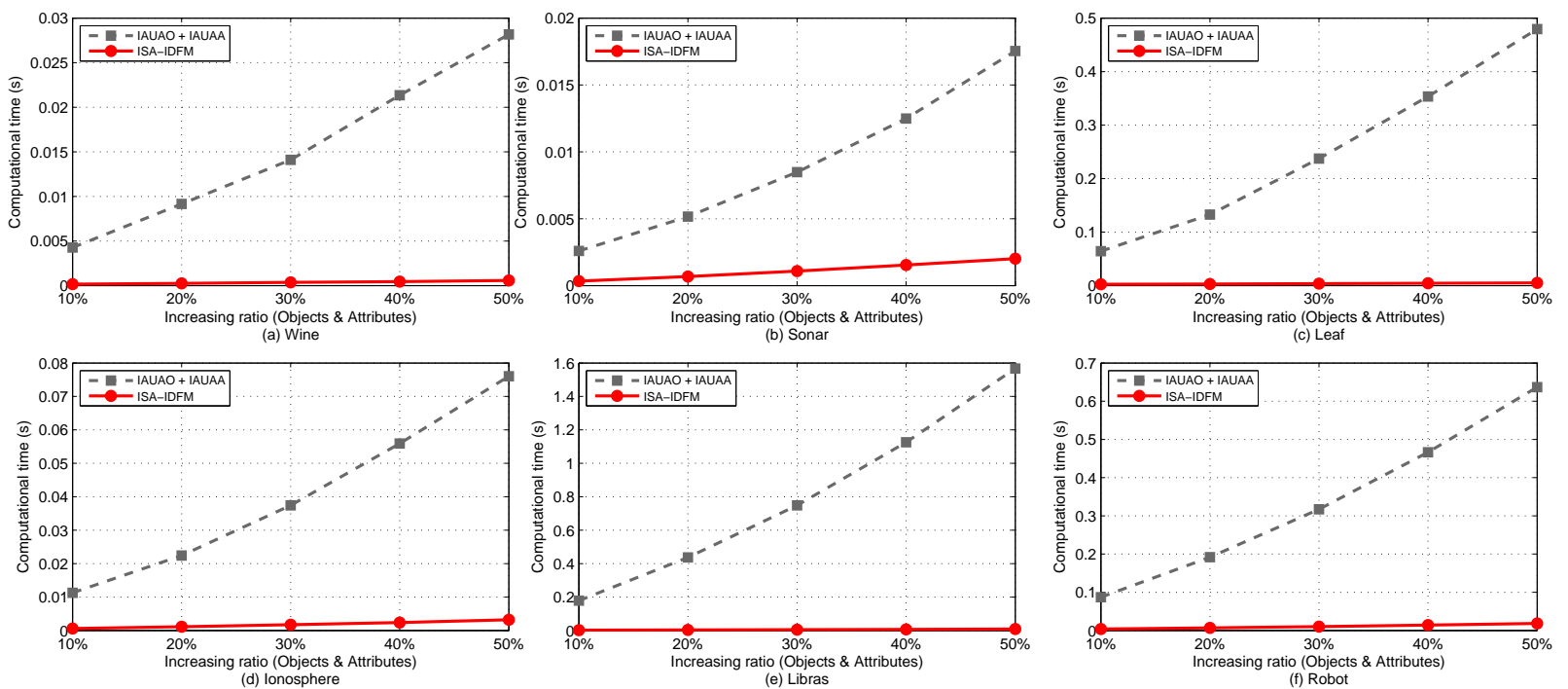

Figure 4: Comparisons of computational time between integrated algorithms and incremental simplified algorithm when objects and attributes increase simultaneously. 
Table 6: Computational time of integrated algorithms and incremental simplified algorithm when objects and attributes increase simultaneously.

\begin{tabular}{|c|c|c|c|c|c|c|c|c|c|c|c|c|}
\hline \multirow[b]{2}{*}{ Data size } & \multicolumn{2}{|c|}{ Wine } & \multicolumn{2}{|c|}{ Sonar } & \multicolumn{2}{|c|}{ Leaf } & \multicolumn{2}{|c|}{ Ionosphere } & \multicolumn{2}{|c|}{ Libras } & \multicolumn{2}{|c|}{ Robot } \\
\hline & Integ. & Incre. & Integ. & Incre. & Integ. & Incre. & Integ. & Incre. & Integ. & Incre. & Integ. & Incre. \\
\hline $60 \%$ & 0.0043 & 0.0002 & 0.0026 & 0.0003 & 0.0643 & 0.0024 & 0.0113 & 0.0006 & 0.1782 & 0.0022 & 0.0872 & 0.0039 \\
\hline $70 \%$ & 0.0092 & 0.0003 & 0.0052 & 0.0007 & 0.1328 & 0.0031 & 0.0224 & 0.0011 & 0.4369 & 0.0035 & 0.1919 & 0.0069 \\
\hline $80 \%$ & 0.0141 & 0.0004 & 0.0085 & 0.0011 & 0.2374 & 0.0037 & 0.0374 & 0.0017 & 0.7481 & 0.005 & 0.3173 & 0.0103 \\
\hline $90 \%$ & 0.0214 & 0.0005 & 0.0125 & 0.0015 & 0.3535 & 0.0044 & 0.0559 & 0.0024 & 1.1258 & 0.0068 & 0.4665 & 0.014 \\
\hline $100 \%$ & 0.0282 & 0.0006 & 0.0175 & 0.002 & 0.4794 & 0.005 & 0.0761 & 0.0032 & 1.5672 & 0.0086 & 0.6367 & 0.0183 \\
\hline
\end{tabular}

\section{Conclusions}

Updating approximations as fast as possible is an important task for processing dynamic data in all rough set models. For the multi-dimensional variation of the ordered information system, in this paper, we presented a novel approach to accelerate updating approximations in DRSA. By analyzing the concepts, principles and parameters of DRSA, we simplified the definitions of P-generalized decision, which neglect unnecessary parameters for the updating. We gave several new notions such as the dominance feature flag, the dominance feature vector and the dominance feature matrix the first time to reflect the dominance relations between objects of their old attributes in an ordered information system. Thus, based on these notions, we employed the incremental learning strategy to avoid lots of repeated comparisons between the old attributes. Then we designed an incremental simplified algorithm, which can efficiently update approximations for the multi-dimensional variation, namely objects and attributes increase simultaneously in an ordered information system. Furthermore, for solving the problem of memory consumption, we presented two implementations based on two different storage schemes for our proposed algorithm. We conducted a series of experiments to evaluate the efficiency of our proposed algorithm. In these experiments, we compared our proposed algorithm with the non-incremental algorithm based on traditional definitions, as well as with the integration of two single-dimensional incremental algorithms. Experimental results showed the advantages of our works. First, when objects and attributes increase simultaneously, our proposed algorithm is always much faster than the non-incremental algorithm and the integration of two single-dimensional algorithms. Second, our proposed algorithm is especially efficient for multi-label data. Third, there is not apparent efficiency difference between the two implementations for our proposed algorithm. So we choose the better one from them to reduce the memory consumption much more. Our future work will focus on dynamic knowledge discovery under other multi-dimensional variations in the ordered information system, such as the simultaneous variation of objects and attribute values.

\section{Acknowledgements}

This work is supported by the National Science Foundation of China (Nos. 61573292, 61572406).

\section{References}

\section{References}

[1] M. G. Augeri, P. Cozzo, S. Greco, Dominance-based rough set approach: An application case study for setting speed limits for vehicles in speed controlled zones, Knowledge-Based Systems, 89 (2015) 288-300.

[2] J. Blaszczynski, S. Greco, R. Slowinski, Marcin. Szelag, Monotonic variable consistency rough set approaches, International Journal of Approximate Reasoning, 50 (7) (2009) 979-999.

[3] H. Chen, T. Li, C. Luo, S. J. Horng, G. Wang, A decision-theoretic rough set approach for dynamic data mining, IEEE Transactions on Fuzzy Systems, 23 (6) (2015) 1958-1970.

[4] H. Chen, T. Li, D. Ruan, J. Lin, C. Hu, A rough-set-based incremental approach for updating approximations under dynamic maintenance environments, IEEE Transactions on Knowledge and Data Engineering, 25 (2) (2013) 274-284.

[5] L. F. Chen, C. T. Tsai, Data mining framework based on rough set theory to improve location selection decisions: A case study of a restaurant chain, Tourism Management, 53 (2016) 197-206. 
[6] Y. Cheng, The incremental method for fast computing the rough fuzzy approximations, Data and Knowledge Engineering, 70 (1) (2011) $84-100$.

[7] K. Dembczynski, S. Greco, R. Slowinski, Rough set approach to multiple criteria classification with imprecise evaluations and assignments, European Journal of Operational Research, 198 (2) (2009) 626-636.

[8] Y. K. Dubey, M. M. Mushrif, K. Mitra, Segmentation of brain MR images using rough set based intuitionistic fuzzy clustering, Biocybernetics and Biomedical Engineering, 36 (2) (2016) 413-426.

[9] S. Greco, B. Matarazzo, R. Slowinski, Rough sets theory for multicriteria decision analysis, European Journal of Operational Research, 129 (1) (2001) 1-47.

[10] S. Greco, B. Matarazzo, R. Slowinski, Rough approximation by dominance relations, International Journal of Intelligent Systems, 17 (2) (2002) 153-171.

[11] S. Greco, B. Matarazzo, R. Slowinski, J. Stefanowski, Variable consistency model of dominance-based rough sets approach, Rough Sets and Current Trends in Computing, Springer Berlin Heidelberg, (2000) 170-181.

[12] Y. C. Hu, Tolerance rough sets for pattern classification using multiple grey single-layer perceptrons, Neurocomputing, 179 (2016) $144-151$.

[13] J. Hu, W. Pedrycz, G. Wang, K. Wang, Rough sets in distributed decision information systems, Knowledge-Based Systems, 94 (2016) 13-22.

[14] B. Huang, H. Li, D. Wei, Dominance-based rough set model in intuitionistic fuzzy information systems, Knowledge-Based Systems, 28 (2012) 115-123.

[15] B. Huang, Y. Zhuang, H. Li, D. Wei, A dominance intuitionistic fuzzy-rough set approach and its applications, Applied Mathematical Modelling, 37 (12) (2013) 7128-7141.

[16] M. Inuiguchi, Y. Yoshioka, Y. Kusunoki, Variable-precision dominance-based rough set approach and attribute reduction, International Journal of Approximate Reasoning, 50 (8) (2009) 1199-1214.

[17] J. Jelonek, E. Lukasik, A. Naganowski, Roman. Slowinski, Inducing jurys preferences in terms of acoustic features of violin sounds, Artificial Intelligence and Soft Computing-ICAISC 2004, Springer Berlin Heidelberg, (2004) 492-497.

[18] J. Karami, A. Alimohammadi, T. Seifouri, Water quality analysis using a variable consistency dominance-based rough set approach, Computers, Environment and Urban Systems, 43 (2014) 25-33.

[19] Y.-C. Ko, H. Fujita, Evidential weights of multiple preferences for competitiveness, Information Sciences 354 (2016) 211-221.

[20] W. Kotlowski, K. Dembczynski, S. Greco, R. Slowinski, Stochastic dominance-based rough set model for ordinal classification, Information Sciences, 178 (21) (2008) 4019-4037.

[21] M. Li, S. Deng, L. Wang, S. Feng, J. Fan, Hierarchical clustering algorithm for categorical data using a probabilistic rough set model, Knowledge-Based Systems, 65 (2014) 60-71.

[22] S. Li, T. Li, D. Liu, Dynamic Maintenance of Approximations in Dominance-Based Rough Set Approach under the Variation of the Object Set, International Journal of Intelligent Systems, 28 (8) (2013) 729-751.

[23] S. Li, T. Li, D. Liu, Incremental updating approximations in dominance-based rough sets approach under the variation of the attribute set, Knowledge-Based Systems, 40 (2013) 17-26.

[24] W. Li, W. Xu, Multigranulation decision-theoretic rough set in ordered information system, Fundamenta Informaticae, 139 (1) (2015) 67-89.

[25] F. Li, M. Ye, X. Chen, An extension to Rough c-means clustering based on decision-theoretic Rough Sets model, International Journal of Approximate Reasoning, 55 (1) (2014) 116-129.

[26] D. Liang, D. Liu, A. Kobina, Three-way group decisions with decision-theoretic rough sets, Information Sciences, 345 (2016) $46-64$.

[27] D. Liang, W. Pedrycz, D. Liu, P. Hu, Three-way decisions based on decision-theoretic rough sets under linguistic assessment with the aid of group decision making, Applied Soft Computing, 29 (2015) 256-269.

[28] J. J. H. Liou, A novel decision rules approach for customer relationship management of the airline market, Expert Systems with Applications, 36 (3) (2009) 4374-4381.

[29] J. J. H. Liou, G. H. Tzeng, A dominance-based rough set approach to customer behavior in the airline market, Information Sciences, 180 (11) (2010) 2230-2238.

[30] J. J. H. Liou, L. Yen, G. H. Tzeng, Using decision rules to achieve mass customization of airline services, European Journal of Operational Research, 205 (3) (2010) 680-686.

[31] D. Liu, T. Li, D. Ruan, J. Zhang, Incremental learning optimization on knowledge discovery in dynamic business intelligent systems, Journal of Global Optimization, 51 (2) (2011) 325-344.

[32] Z. Lu, Z. Qin, Y. Zhang, J. Fang, A fast feature selection approach based on rough set boundary regions, Pattern Recognition Letters, 36 (2014) 81-88.

[33] C. Luo, T. Li, Z. Yi, H. Fujita, Matrix approach to decision-theoretic rough sets for evolving data, Knowledge-Based Systems, 99 (2016) 123-134.

[34] G. Z. Luo, X. B. Yang, Limited Dominance-based Rough Set Model and Knowledge Reductions in Incomplete Decision System, Journal of Information Science and Engineering, 26 (6) (2010) 2199-2211.

[35] J. Meng, J. Zhang, R. Li, Y. Luan, Gene selection using rough set based on neighborhood for the analysis of plant stress response, Applied Soft Computing, 25 (2014) 51-63.

[36] I. K. Park, G. S. Choi, Rough set approach for clustering categorical data using information-theoretic dependency measure, Information Systems, 48 (2015) 289-295.

[37] Z. Pawlak, Rough sets, International Journal of Computer and Information Sciences, 11 (5) (1982) 341-356.

[38] G. Peters, S. Poon, Analyzing IT business valuesCa dominance based rough sets approach perspective, Expert Systems with Applications, 38 (9) (2011) 11120-11128

[39] A. Phophalia, A. Rajwade, S. K. Mitra, Rough set based image denoising for brain MR images, Signal Processing, 103 (2014) 24-35.

[40] M. S. Raza, U. Qamar, An incremental dependency calculation technique for feature selection using rough sets, Information Sciences, 343 (2016) 41-65.

[41] R. Zhang, B. Liu, S. Liu, A multi-attribute auction model by dominance based rough sets approach, Computer Science and Information Systems, 7 (4) (2010) 843-858. 
[42] W. Shu, H. Shen, Incremental feature selection based on rough set in dynamic incomplete data, Pattern Recognition, 47 (12) (2014) 3890 3906.

[43] R. Susmaga, Reducts and constructs in classic and dominance-based rough sets approach, Information Sciences, 271 (2014) 45-64.

[44] X. Yang, J. Xie, X. Song, J. Yang, Credible rules in incomplete decision system based on descriptors, Knowledge-Based Systems, 22 (1) (2009) 8-17.

[45] X. Yang, J. Yang, C. Wu, D. Yu, Dominance-based rough set approach and knowledge reductions in incomplete ordered information system, Information Sciences, 178 (4) (2008) 1219-1234.

[46] X. Yang, D. Yu, J. Yang, L. Wei, Dominance-based rough set approach to incomplete interval-valued information system, Data and Knowledge Engineering, 68 (11) (2009) 1331-1347.

[47] H. Yu, Z. Liu, G. Wang, An automatic method to determine the number of clusters using decision-theoretic rough set, International Journal of Approximate Reasoning, 55 (1) (2014) 101-115.

[48] H. Y. Zhang, Y. Leung, L. Zhou, Variable-precision-dominance-based rough set approach to interval-valued information systems, Information Sciences, 244 (2013) 75-91.

[49] J. Zhang, J. S. Wong, Y. Pan, T. Li, A parallel matrix-based method for computing approximations in incomplete information systems, IEEE Transactions on Knowledge and Data Engineering, 27 (2) (2015) 326-339.

[50] X. Zhang, D. Miao, C. Liu, M. Le, Constructive methods of rough approximation operators and multigranulation rough sets, KnowledgeBased Systems, 91 (2016) 114-125. 